\title{
Self-Adversarial Training incorporating Forgery Attention for Image Forgery Localization
}

\author{
Long Zhuo, Shunquan Tan*, Senior Member, IEEE, Bin Li, Senior Member, IEEE, and Jiwu Huang, Fellow, IEEE
}

\begin{abstract}
Image editing techniques enable people to modify the content of an image without leaving visual traces and thus may cause serious security risks. Hence the detection and localization of these forgeries become quite necessary and challenging. Furthermore, unlike other tasks with extensive data, there is usually a lack of annotated forged images for training due to annotation difficulties. In this paper, we propose a self-adversarial training strategy and a reliable coarse-to-fine network that utilizes a self-attention mechanism to localize forged regions in forgery images. The self-attention module is based on a ChannelWise High Pass Filter block (CW-HPF). CW-HPF leverages interchannel relationships of features and extracts noise features by high pass filters. Based on the CW-HPF, a self-attention mechanism, called forgery attention, is proposed to capture rich contextual dependencies of intrinsic inconsistency extracted from tampered regions. Specifically, we append two types of attention modules on top of $\mathrm{CW}-\mathrm{HPF}$ respectively to model internal interdependencies in spatial dimension and external dependencies among channels. We exploit a coarse-to-fine network to enhance the noise inconsistency between original and tampered regions. More importantly, to address the issue of insufficient training data, we design a self-adversarial training strategy that expands training data dynamically to achieve more robust performance. Specifically, in each training iteration, we perform adversarial attacks against our network to generate adversarial examples and train our model on them. The proposed method is based on the assumption of content-changed manipulations. Extensive experimental results demonstrate that our proposed algorithm steadily outperforms state-of-the-art methods by a clear margin in different benchmark datasets.
\end{abstract}

Index Terms-Forgery localization, forgery attention, coarseto-fine network, self-adversarial training.

\section{INTRODUCTION}

$\mathbf{T}$ HE prevalence of digital image forgery is negatively affecting our lives, such as Internet rumors, insurance fraud, fake news, and even academic cheating [1]. Ghanim and Nabil [2] revealed that image forgery might cause huge financial loss. Bik et al. [3] estimated that there were $3.8 \%$ of 20,621 papers containing problematic figures with potential deliberate manipulation in biomedical research publications.

*Corresponding author: Shunquan Tan.

All of the members are with the Guangdong Key Laboratory of Intelligent Information Processing, Shenzhen Key Laboratory of Media Security, Guangdong Laboratory of Artificial Intelligence and Digital Economy (SZ), Shenzhen Institute of Artificial Intelligence and Robotics for Society, China (email: zhuolong@email.szu.edu.cn; tansq, libin,jwhuang@szu.edu.cn).

S. Tan is with College of Computer Science and Software Engineering, Shenzhen University, Shenzhen 518060, China.

This work was supported in part by NSFC (U19B2022, 61772349, 61872244, 62072313, 61806131, 61802262), Guangdong Basic and Applied Basic Research Foundation (2019B151502001), and Shenzhen R\&D Program (JCYJ20200109105008228, 20200813110043002). This work was also supported in part by Alibaba Group through Alibaba Innovative Research (AIR) Program.
Yet, the majority of image forgeries cases have not been detected [4] due to the fact that image manipulation is very difficult to detect, and tampered images are commonly indistinguishable from original images by naked eyes. With the automatic generation techniques (e.g., inpainting with generative adversarial networks) and popular image editing tools (such as Photoshop ${ }^{\circledR}$, After Effects Pro ${ }^{\circledR}$, and the opensource software GIMP), it is easy to generate forged images at low cost. The localization of tampered regions turns out to be very tricky. Therefore, as tampered images grow at an enormous rate, it is necessary to develop new algorithms against image forgery.

Media forensics has been developed to detect image forgeries for many years. However, few early works focused on forgery detection at pixel level. The researchers made great efforts in classifying whether an image has been tampered or not with traditional features, like wavelet transform feature [5], point matching [6], and self-consistency [7]. Furthermore, most of them detect only one specific type of manipulation, such as splicing [7], [8], copy-move [9], and removal [10]. Therefore, real-world media forensics is desperate for a new generation of algorithms that can obtain more refined results at the pixel level, as well as the detection of general manipulations.

However, it is challenging to localize multiple image forgeries at the pixel level since well-tampered images leave few visual traces. Conventional detection methods based on manually constructed statistical features rely heavily on the domain knowledge of human experts.

Forgery localization task aims to localize the contentchanged tampering techniques, including splicing, copy-move and removal, since content-changed techniques may cause serious misunderstandings while tampering with unchanged content does not change the semantics without causing misunderstandings. Please note that non-content-changed manipulations, such as Gaussian blur and JPEG compression, are excluded in the literature of image forgery localization, since those manipulations do not affect the semantic information expressed in the image scene. Therefore, all of the existing image forgery localization approaches [4], [11], [12], [13], [14], [15] only focus on three common semantic forgeries, i.e. image splicing, copy-move and removal. Examples of the three common semantic forgeries are shown in Fig. 11 Our work follows this assumption.

Some early methods focused on low-level tampering artifacts in image forgeries, such as JPEG compression [16] and CFA color array analysis [17].

Recent works, including Mantra-Net [4], RGB-N [11], J- 


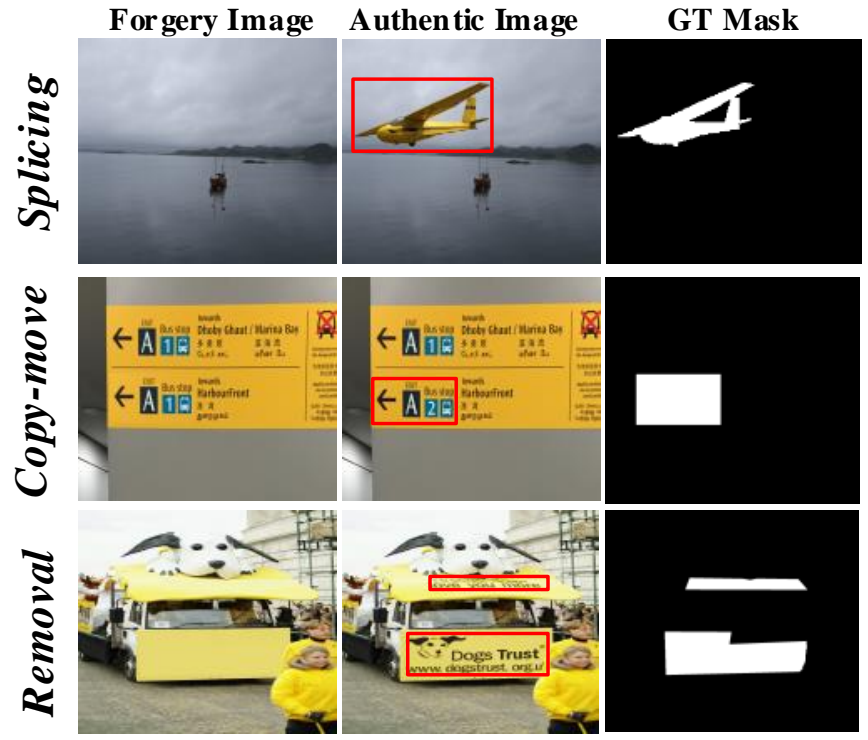

Fig. 1. Examples of three common kinds of image forgeries, i.e., image splicing, copy-move and removal. Compared to authentic images, forgery images change the semantic meanings significantly. The tampered regions are highlighted with red rectangles in the authentic images. However, they are so secretive that it is hard for human eyes to point out them.

LSTM [12], H-LSTM [13], have proposed general and endto-end solutions for image forgery localization using deep learning-based approaches. Both Wu et al. [4] and Zhou et al. [11] borrowed three high pass filters from Steganalysis Rich Model (SRM) [18] prior to their end-to-end framework to discover noise inconsistency between authentic and tampered regions. Specifically, Mantra-Net [4] is a joint system that predicts manipulation traces for both image manipulation classification and forgery localization. It employs a VGGbased [19] feature extractor to capture location information. Zhou [11] presented a two-stream network which consists of a regular RGB-based faster R-CNN stream and a parallel stream generated with SRM based noise extractor. The drawback of RGB-N is that it can only mark tampered regions with rectangular boxes due to the R-CNN structure. In the meantime, Bappy et al. [12] applied an LSTM-based patch comparison method (J-LSTM) to detect the boundary of tampered and authentic patches and further presented a separate encoderdecoder structure (H-LSTM) [13] to improve the performance.

More recently, some approaches [20], [21], [14] used attention modules to focus on important regions in a target image. In the most recent one of them, $\mathrm{Hu}$ et al. [14] proposed a so-called SPAN model (Spatial Pyramid Attention Network), which is also selected for comparison in our work. The problem of all of the mentioned attention-module based methods is that their key components are directly borrowed from object detection, and consequently are not well incorporated in forgery localization. For instance, their attention modules are object-sensitive and only pay attention to salient objects in an image. Since quite a few tampered regions are not objects in real scenarios, traditional object attention modules might fail to guide networks' attention to tampered regions. Therefore, it is important to construct a novel network structure which can adapt attention mechanisms to forgery localization.

Furthermore, all state-of-the-art end-to-end solutions, e.g., SPAN [14], Mantra-Net [4], and RGB-N [11], have adopted normal data augmentation techniques, such as image flipping and rotation, to obtain twice or more number of training samples to avoid model over-fitting. However, with ultra-limited training samples in this field, additional samples obtained with normal data augmentation are still limited. It is necessary to develop a new training strategy for forgery localization to increase sample numbers by one or two orders of magnitude.

To address the above issues, we propose a novel framework and a self-adversarial training strategy toward the goal of localizing forged regions in images precisely. The proposed network involves a Channel-Wise High Pass Filter (CW-HPF) block and a forgery attention mechanism. Furthermore, we introduce the coarse-to-fine architecture to enhance network representations.

Specifically, CW-HPF splits an input feature map into channels. It then performs convolution initialized by three high pass filters, called HPF-Conv, on each channel to extract noise features, respectively. The HPF-Conv output is fused to uncover the inter-channel relationships of these noise features. The channel-wise architecture of CW-HPF is motivated by WISERNet [22], a powerful deep steganalysis model using inter-channel information.

On top of CW-HPF, we propose forgery attention, a novel attention mechanism considering forgery localization task. Inspired by the dual attention module [23], forgery attention aims to capture noise feature dependencies in both spatial and channel dimensions. In the spatial dimension, we extract spatial dependencies of noise features in every pixel pair of feature maps. Similarly, in the channel dimension, we capture channel dependencies of noise features among channel pairs. The outputs of two attentive dimensions are fused to enhance feature representations. Forgery attention enables our model to adaptively integrate local noise features with their global dependencies.

To alleviate the problem of limited training data, we present a novel Self-Adversarial Training (SAT) strategy for dynamical data augmentation. SAT exploits adversarial attacks [24] in every training iteration and generates new training data dynamically, which guides our model to defend from adversarial attacks and achieve more robust performance. Different from traditional data augmentation, our SAT can provide inexhaustible new adversarial training data according to model updating.

As for the experiments, the notable public datasets for forgery localization, including DEFACTO [25], NIST [26], Columbia [25], COVERAGE [27], CASIA [9] and PSdataset [15], are established on the content-changed manipulations. Therefore, the assumption of our experiments is that the forgery localization models aim to localize the contentchanged regions. Based on this assumption, extensive experiments demonstrate that our approach has achieved state-ofthe-art results on all primary benchmark datasets.

To be summarized, our main contribution is to propose a novel image deep-learning based forgery localization framework that organically combines the domain knowledge of 
multimedia security and image recognition. The novelty of this work can be explicitly highlighted as follows: 1) we propose Forgery attention, a novel attention mechanism for forgery localization, that fuses domain knowledge well-established in multimedia security into attention mechanism, and makes a combination of channel-wise and spatial dependencies. 2) A new training strategy, namely SAT, is first introduced to alleviates the problem of the lack of training data for improving the localization performance and the model robustness. 3) we move a further step for HPF layer and present CW-HPF that exploits the inter-channel relationships and generates more precise noise features.

The remainder of the paper is organized as follows. In Section $[$ II] we present our proposed approach in details. Then, we show the results of our extensive experiments in Section III. Finally, we conclude this paper in Section IV

\section{PROPOSED APPROACH}

In this section, we present the general framework of our proposed network as well as self-adversarial training strategy and then formulate our approach.

\section{A. Overview}

Given an image, we aim to localize its tampered regions at the pixel level. The general framework of our proposed model is illustrated in Fig. 22 Furthermore, we propose selfadversarial training to promote the robustness of the network since forged training samples are ultra-limited, whose details will be given in Sect. II-E Overall, we construct a coarse-tofine network containing a coarse output mask and a refined output mask. Next, we propose a two-phase training strategy called self-adversarial training strategy to perform our training process, where our network is trained using the original inputoutput pairs commonly in the first phase and then trained using new input-output pairs with adversarial samples in the second phase.

Our network has a coarse-to-fine architecture. It is composed of two sub-networks, i.e., a coarse net and a refined net. The coarse net is fed with a forged image $I_{F}$ of size $H \times W \times 3$ and outputs a coarse prediction mask of size $H \times W$ and a feature map $\hat{I_{M_{1}}}$ of size $H \times W \times k$, where $k$ is set to 16 because the output of the last block outputs a feature map with 16 channels. The feature map ${\hat{M_{M}}}_{1}$ is fed into the refined net that predicts a refined prediction mask $I_{M}$ of size $H \times W$. Note that $I_{M_{1}}$ and $I_{M}$ come from two output convolution layers with a kernel size of $7 \times 7$, activated by a sigmoid function. $\hat{I_{M_{1}}}$ aims to deliver complete feature information to the refined net, which enables the refined net to be optimized along with the features. The predicted masks mark tampered regions in white and leave the rest in black. We select the refined mask $I_{M}$ as the final result.

As illustrated in Fig. 2, there are three key components in the coarse net, including a CW-HPF block, multiple VGGstyle blocks, and a series of dilated convolutional layers. The refined net adopts similar structure, but with a forgery attention module attached.

\section{B. CW-HPF Block}

HPF layer widely used in image steganalysis [18] has been adopted in forgery localization [4], [11]. The intuition behind the usage of high-pass filters is that tampering traces are generally manifested in the middle and high-frequency sub-bands of the tampered image. However, prior works have neglected the relationships among channels of images, which may yield many unnecessary noise features. To extract more accurate noise features, we leverage the inter-channel information to enhance the noise inconsistency between tampered and pristine regions.WISERNet [22], our prior work in the field of image steganalysis has proved in theory that noise features extracted by channel-wise high pass filters could enlarge the slight difference between the authentic images and the manipulated images with tiny stego noises. However, WISERNet only uses the channel-wise high pass filters with fixed weights and three $\mathrm{R}-\mathrm{G}-\mathrm{B}$ color channels as input in a bottom pre-processing layer.

Move a step further, in our proposed framework we have introduced CW-HPF which can be fed with arbitrary input channels and can be put in arbitrary positions/branches of our framework.

Fig. 3 shows the details of the CW-HPF block. CW-HPF takes a feature map of size $H \times W \times C$ and outputs a noise feature map of size $H \times W \times 3 C$. Firstly, the input feature map is converted to a feature map set $\mathcal{S}$ with $C$ feature maps. $\mathcal{S}_{i}$ represents the $i$-th channel of the input feature map. We define $\mathcal{S}$ as $\mathcal{S}=\left\{\mathcal{S}_{1}, \mathcal{S}_{2}, \mathcal{S}_{3}, \ldots, \mathcal{S}_{C}\right\}$. We employ three high pass filters originated from SRM [18] to initialize a convolution layer of size $5 \times 5 \times 3$, which we call HPF-Conv. As shown in Fig. 4 the selected high pass filters involve a KB filter, a KV filter and a first order kernel, which are the same as RGB-N [11]. Unlike steganalysis, the forgery localization task needs only three high pass filters from thirty steganalysis rich model filters to achieve decent performance and save computing resources [11]. We apply HPF-Conv to perform convolution with each element of the feature map set $\mathcal{S}$, and the results are concatenated to produce noise features of size $H \times W \times 3 C$.

Our motivation is that the slight high-frequency differences between the authentic images and the manipulated images ought to be passed on through cascaded layers. As a result, CW-HPF can be used in different parts of our proposed framework to extract more precise noise features by leveraging the inter-channel relationships, and thus improves the overall performance.

CW-HPF in this work is unlearnable, and the filters are handcrafted.

\section{Forgery Attention}

Attention mechanism enables a neural network focus on important regions of its feature representations. Attention helps it build input-aware connections to focus more on meaningful regions by replacing fixed weights with input dependent weights. Attention mechanism has been heavily involved in different application fields of deep-learning frameworks, such 


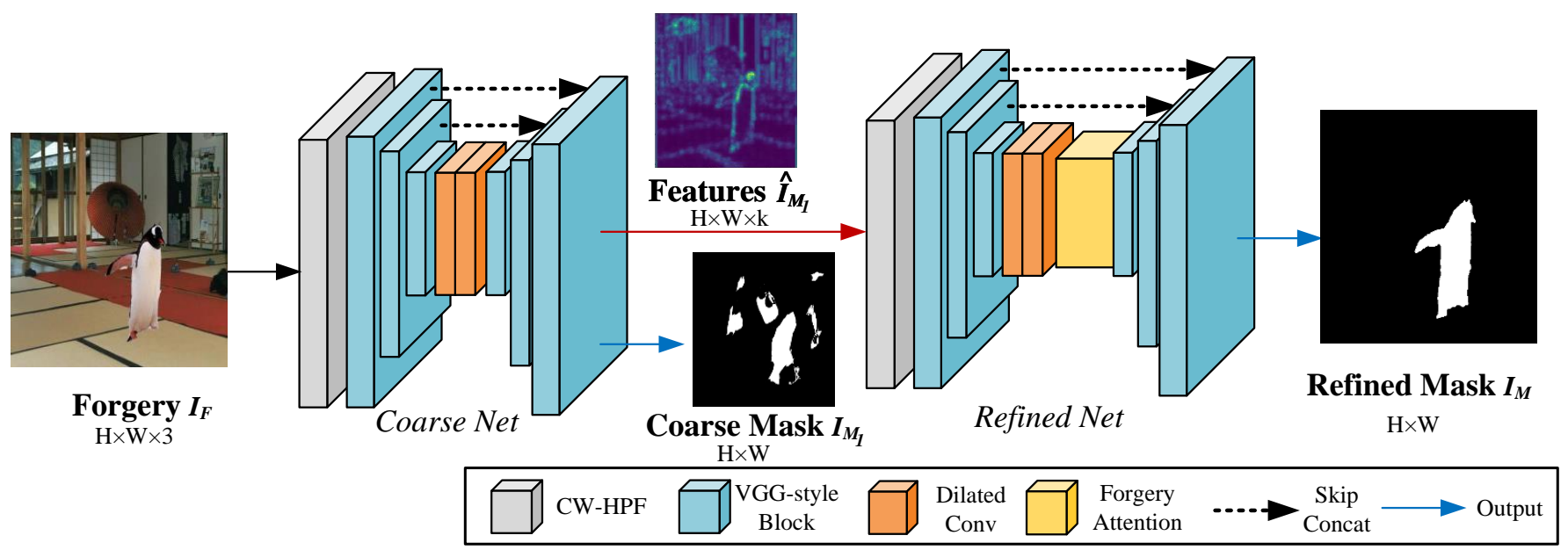

Fig. 2. Illustration of our proposed coarse-to-fine network for forgery localization task. It is composed of two sub-networks, namely coarse-net and refined-net. The coarse-net takes a forged image $I_{F}$ as input and predicts a coarse mask $I_{M_{1}}$ and a feature map $I_{M_{1}}$. The refined-net takes $\hat{I}_{M_{1}}$ and predicts a refined mask $I_{M}$.

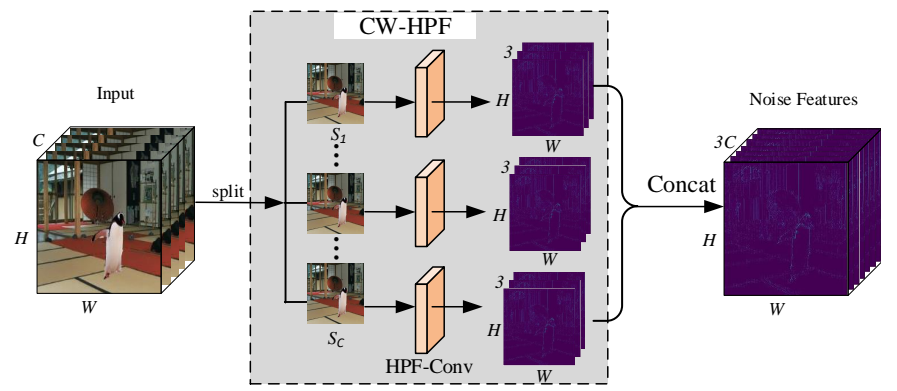

Fig. 3. Details of the Channel-Wise High Pass Filter (CW-HPF) block.

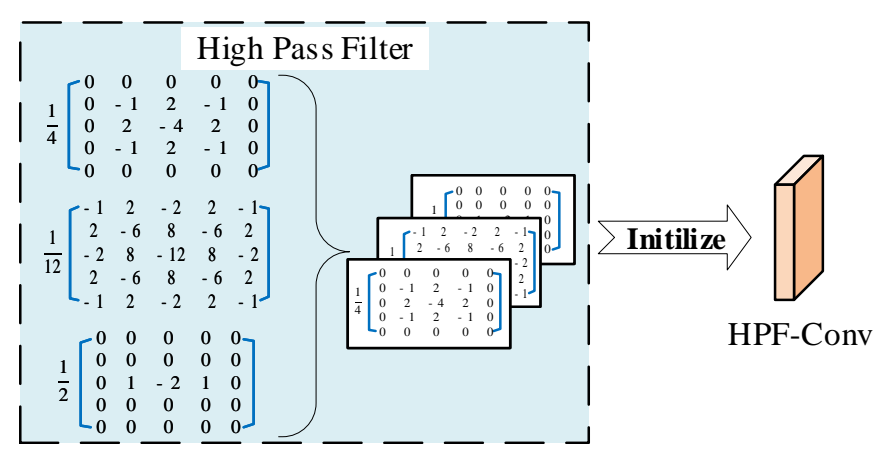

Fig. 4. Details of the High Pass Filter Convolution (HPF-Conv) layer.

as machine translation [28], image captioning [29] and object detection [30].

SPAN [14] borrowed a single spatial attention mechanism from image recognition, with two downsides: 1) it ignores the color-channel-wise dependencies, which are important for forgery localization of true color images with multiple color channels; 2) it only focuses on salient objects on the scene while quite a few tampered regions are not with salient objects.

To overcome the downsides of normal attention mechanism in forgery localization, we have fused domain knowledge well established in multimedia security into attention mechanism, as well as made a combination of channel-wise and spatial de- pendencies. Here, we propose a forgery attention mechanism to focus on tampered traces instead of salient objects in order to make it adapt to forgery localization tasks. The details of our forgery attention are formulated in Algorithm 1 .

We construct two attention branches to obtain global noise attention features, as shown in Fig. 5. We feed local features $F \in \mathbb{R}^{H \times W \times C}$ generated by the dilated convolutional module of the refined net into two CW-HPF-based parallel attention branches, namely a spatial attention branch and a channel attention branch.

Our motivation is that with our proposed forgery attention module, the extracted attention features can provide the similarity map of noise features in both channel and spatial dimensions. It can reflect long-term contextual information in the noise domain since any two positions with similar noise features can contribute mutual improvement regardless of their distance in both spatial dimension and channel dimension.

The spatial attention branch generates a Spatial-dimension Attention Feature map ( denoted as $\mathrm{SAF}_{N}$ ). It draws the spatial relationship between pairwise positions of the noise features. We update the features of each position by aggregating noise features of all positions with a weighted sum, where the weights are calculated by the similarities of the noise features between the corresponding two positions. For any two positions with similar noise features in the spatial dimension, they can contribute to mutual improvement.

Specifically, in the spatial dimension, the input $F$ is fed into the CW-HPF block, followed by a convolution layer, to extract noise features. Three convolution layers are applied over the noise feature map in parallel to generate three feature maps $F_{1}, F_{2}, F_{3} \in \mathbb{R}^{H \times W \times C}$, respectively. $F_{1}, F_{2}$ and $F_{3}$ are then reshaped to two-dimensional feature maps $F_{1}^{\prime}, F_{2}^{\prime}$ and $F_{3}^{\prime}$, each of which belongs to $\mathbb{R}^{(H \times W) \times C} . F_{1}^{\prime}$ is further transposed to $F_{1}^{\prime \top} \in \mathbb{R}^{C \times(H \times W)}$. Matrix cross product of $F_{2}^{\prime}$ and $F_{1}^{\prime \top}$ is performed to calculate the distances between different positions. Here, the result of the product measures the impact of $i^{\text {th }}$ position on $j^{\text {th }}$ position, where $(i, j)$ in $((H \times W),(H \times W))$. The matrix cross product is further 


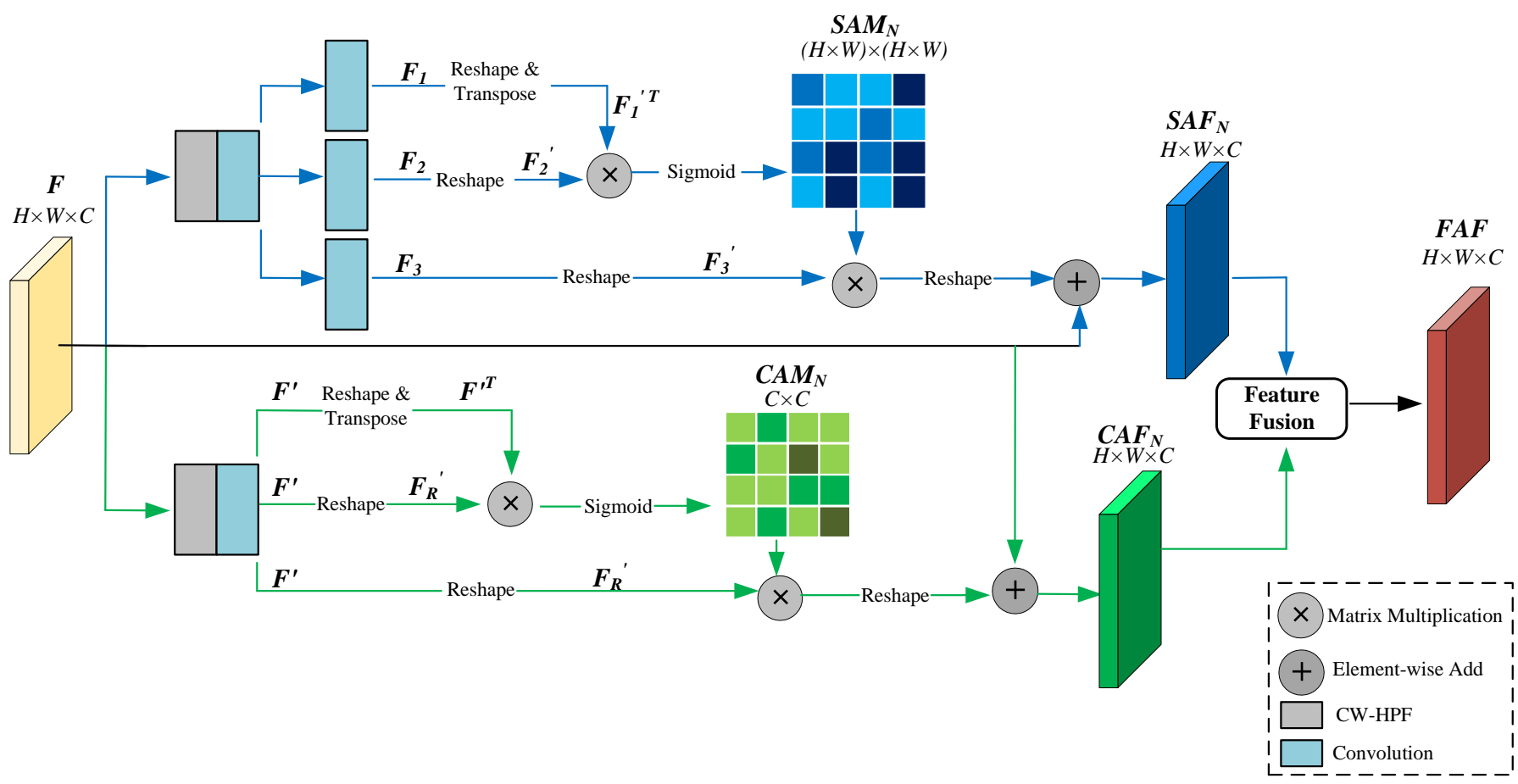

Fig. 5. The details of forgery attention. There are two parallel branches in forgery attention. The top one is the spatial attention branch, and the bottom one is the channel attention branch. Note that the cells with different darkness in $\mathrm{SAM}_{N}$ and $\mathrm{CAM}_{N}$ indicate the amount of attention paid to the noise features of these regions.

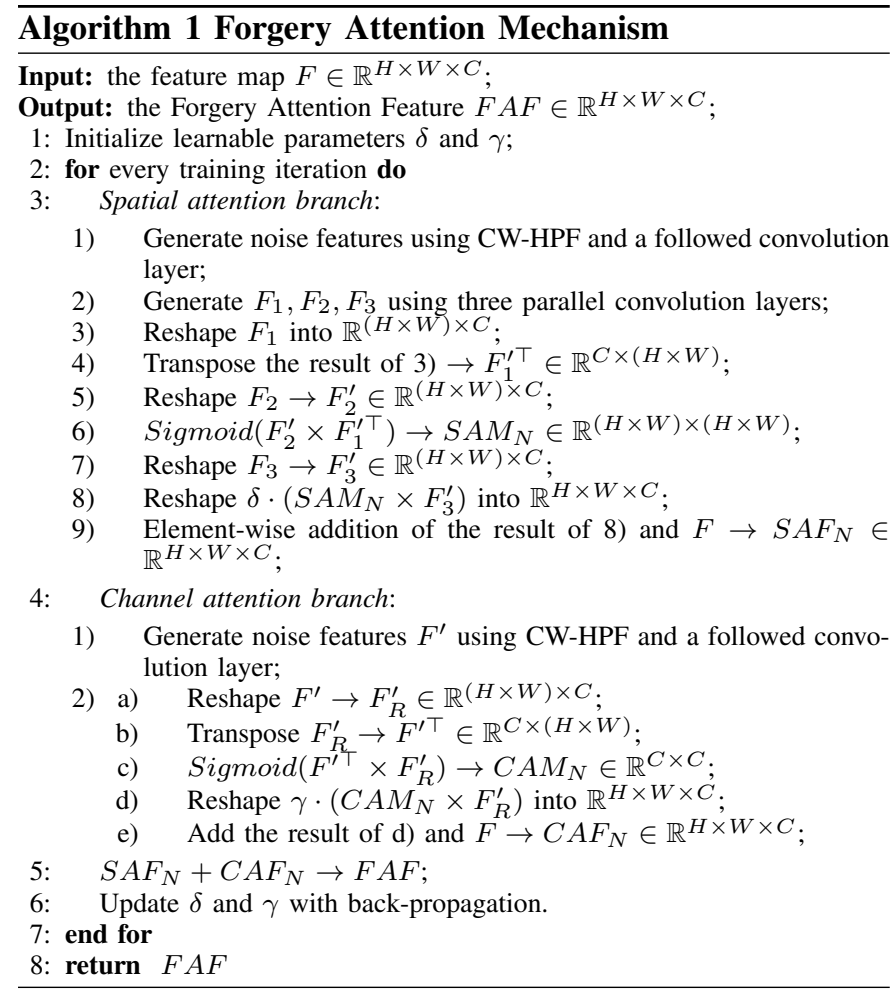

fed to a Sigmoid activation to get the Spatial Attention Matrix of noise features $\mathrm{SAM}_{N} \in \mathbb{R}^{(H \times W) \times(H \times W)}$. $\mathrm{SAM}_{N}$ describes the similarity between any two different positions in the spatial dimension of the noise feature map. According to [23], the more similarity between two positions in the noise feature map is, the greater the correlation between two points in $F$ becomes. $\mathrm{SAM}_{N}$ is then multiplied with $F_{3}$, and the result is reshaped back to $H \times W \times C$. Finally, a learnable scaling factor $\gamma$ is multiplied with the result of the last step and then is added with the input features $F$ to generate $\mathrm{SAF}_{N} \in \mathbb{R}^{H \times W \times C}$. It is formulated as follows:

$$
\begin{aligned}
\operatorname{SAF}_{N} & =\gamma\left(\operatorname{SAM}_{N} \times F_{3}\right)+F \\
& =\gamma\left(\operatorname{sigmoid}\left(F_{1}^{\top} \times F_{2}^{\prime}\right) \times F_{3}^{\prime}\right)+F,
\end{aligned}
$$

where we initialize $\gamma$ as 0 and update it with back-propagation learning.

Meanwhile, the channel attention branch generates a Channel-dimension Attention Feature map ( denoted as $\mathrm{CAF}_{N}$ ) to model the channel relationship between any two channels of noise features. We update each channel feature map with a weighted sum of all channel feature maps.

The calculation in the channel dimension is similar to that in the spatial dimension. Firstly, a CW-HPF and a convolution layer are used to generate the noise features $F^{\prime}$ of input $F$, where $F^{\prime} \in \mathbb{R}^{H \times W \times C}$. $F^{\prime}$ is reshaped to $F_{R}^{\prime} \in \mathbb{R}^{(H \times W) \times C}$. $F_{R}^{\prime}$ is then performed matrix cross product with $F^{\prime \top}$. A Sigmoid activation is applied to calculate the Channel Attention Matrix $\mathrm{CAM}_{N} \in \mathbb{R}^{C \times C}$ of noise features. Like $\mathrm{SAM}_{N}$, $\mathrm{CAM}_{N}$ describes the similarity between any two different positions in the channel dimension of the noise feature map. Then $F_{R}^{\prime}$ is performed matrix cross product with $\mathrm{CAM}_{N}$, whose result is reshaped to $H \times W \times C$. Finally, the matrix cross product result is multiplied by another learnable scaling factor $\delta$, and the multiplication result is added with the input $F$ 
to obtain $\mathrm{CAF}_{N} \in \mathbb{R}^{H \times W \times C}$ :

$$
\begin{aligned}
\operatorname{CAF}_{N} & =\delta\left(\operatorname{CAM}_{N} \times F_{R}^{\prime}\right)+F, \\
& =\delta\left(\operatorname{sigmoid}\left(F^{\prime \top} \times F_{R}^{\prime}\right) \times F_{R}\right)+F,
\end{aligned}
$$

where $\delta$ starts from 0 and gradually adjusts to assign more weight during training.

The introduction of the two learnable scaling factors, namely $\gamma$ and $\delta$, during the training procedure of $\operatorname{SAF}_{N}$ and $\mathrm{CAF}_{N}$ is borrowed from [23], in order to enhance network representation. In addition, please note that there are only two classes (i.e., forged or not) in the forgery localization task, and usually prediction of every pixel ranges from 0 to 1 . Therefore, in forgery attention, $\mathrm{SAF}_{N}$ and $\mathrm{CAF}_{N}$ are generated with Sigmoid activation rather than traditional Softmax function since the value of Sigmoid activation is confined in $[0,1]$.

After that, $\mathrm{SAF}_{N}$ and $\mathrm{CAF}_{N}$ are then fused to obtain the Forgery Attention Features (FAF). In particular, an elementwise addition is performed on $\mathrm{SAF}_{N}$ and $\mathrm{CAF}_{N}$, of which the result is fed into a convolution layer to generate FAF. As a result, FAF provides the similarity map of noise features in both channel and spatial dimensions. It reflects long-term contextual information in the noise domain because any two positions with similar noise features can contribute mutual improvement regardless of their distance in both spatial dimension and channel dimension.

\section{VGG Block and Dilated Convolutional Block}

The architecture of VGG blocks originates from MantraNet [4]. Each VGG block contains three or four stacked convolution layers with kernel size $3 \times 3$. In coarse net, the VGG modules are denoted as $\mathcal{V}_{i}(f)(i \in\{1,2,3,4,5\})$, where $f$ denotes the input features and $\mathcal{V}_{i}$ denotes VGG block. $\mathcal{V}_{i}$ performs encoding when $i=1,2,3$ while performs decoding when $i=4,5$. The VGG blocks are of size $32 \times 2^{i}$ when $i=1,2,3$, and size $32 \times 2^{5-i}$ when $i=4,5$. Max pooling layers follow $\mathcal{V}_{1}(f)$ and $\mathcal{V}_{2}(f)$ to down-sample the features, and the output of them is skip-concatenated with that of $\mathcal{V}_{5}(f)$ and $\mathcal{V}_{4}(f)$, respectively. $\mathcal{V}_{4}(f)$ and $\mathcal{V}_{5}(f)$ are followed by up-sampling layers to restore the size of feature maps.

Zhuang et al. [15] has pointed out that for forgery localization task the deep-learning framework needs larger receptive fields to avoid learning features from narrow local regions. Thus following their approach, four dilated convolution layers are applied to inflate the kernels by inserting zeros between kernel elements with different dilation rates for extracting features with larger receptive fields. The dilated convolution layers are then used to bridge the encoders and the corresponding decoders in both the coarse net and the refined net. Specifically, the dilation rates in the four dilated convolution layers are $2,4,8$, and 16 , respectively.

\section{E. Self-Adversarial Training}

Frankly speaking, deep-learning based models are all training data hungry. It is a huge challenge to train a deep-learning based framework in a scenario with limited training samples, such as image forgery localization. Data augmentation

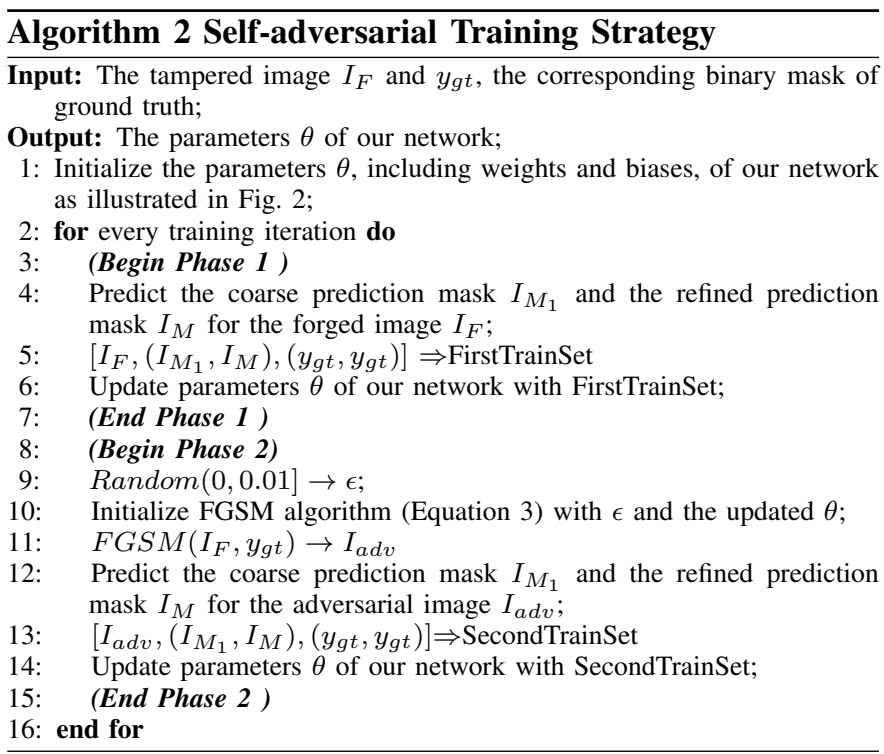

techniques can alleviate this issue. However, the common data augmentation techniques, such as image flipping and rotation, used in existing forgery localization methods (e.g. Mantra-Net [4] and SPAN [14]) do not utilize the feedback of the target deep-learning based framework in training samples augmentation.

Our proposed SAT strategy can realize training samples augmentation with endless supply of adversarial samples generated with the latest gradients of the target deep-learning model in every training iteration.

The motivation behind SAT is that the detection model can easily over-adapt to the texture features of the image datasets it is trained on, since the tampering noise is very subtle. SAT not only increases the robustness of the model with adversarial attack during training, but also improves its performance by providing training data dynamically. In the field of object detection, YOLO v4 [31] has used self-adversarial training to augment the training data and achieve better performance. We first attempt for applying self-adversarial training strategy and making experimental analysis of it for forgery localization.

In the first training phase, like the traditional training process, our model is trained on the forged image $I_{F} \in \mathbb{R}^{H \times W \times C}$ and its corresponding ground-truth mask $y_{g t}$.

In the second training phase, an adversarial image $I_{\text {adv }}$ from $I_{F}$ is firstly generated with the Fast Gradient Sign Method (FGSM) [24], a fast and famous adversarial attack method. Due to the fast attack speed of FGSM, we apply it instead of other adversarial attacks as our attack method. Other well-known adversarial attack algorithms, such as BIM [32] and MI-FGSM [33], spend more time on attacking an image. Specifically, BIM takes 0.94 second, MI-FGSM takes $1.13 \mathrm{sec}-$ ond, while FGSM only take 0.2 second to perform adversarial attack on an image. FGSM attacks the latest gradients of our model to generate an adversarial example $I_{\text {adv }} \in \mathbb{R}^{H \times W \times C}$, which can be formulated as follows:

$$
I_{\mathrm{adv}}=I_{F}+\epsilon \cdot \operatorname{sign}\left(\nabla_{I_{F}} L\left(\theta, I_{F}, y_{g t}\right)\right),
$$

where $\theta$ denotes the current parameters of our model, $\nabla_{I_{F}}$ 

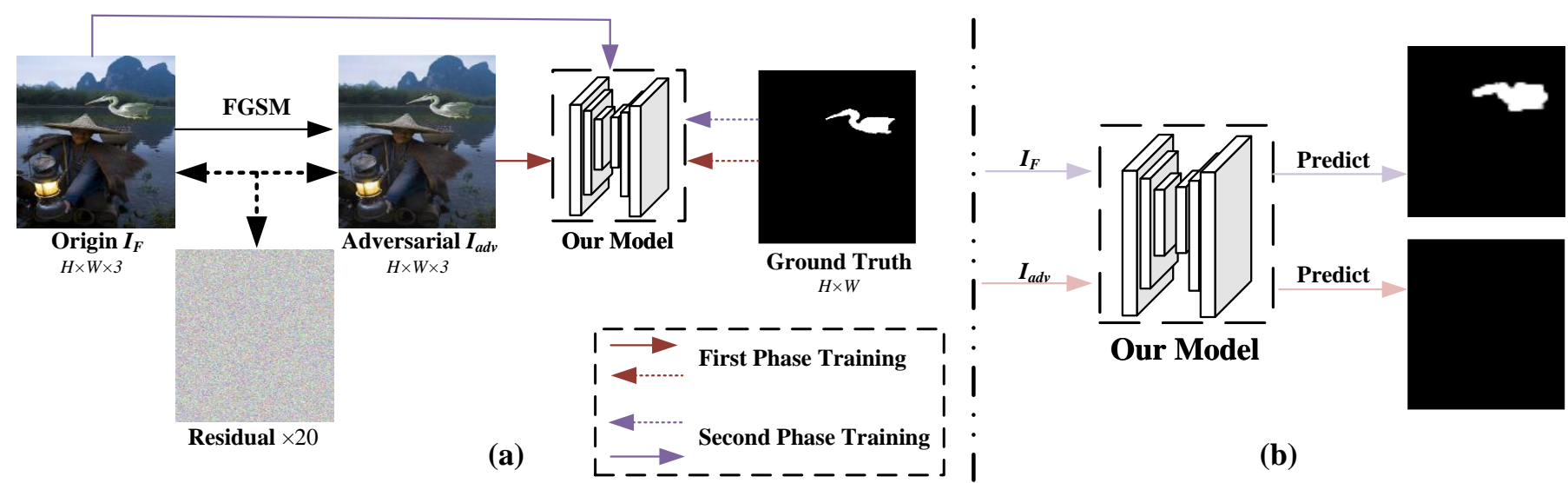

(b)

Fig. 6. Illustration of our proposed SAT. It trains our model in two phases. Fig. (a) shows the training procedure of SAT. Values of the residual map between $I_{F}$ and $I_{a d v}$ are multiplied by 20 for better visualization. Fig. (b) shows different responses of our model prior to the second phase, when it is fed with $I_{F}$ or fed with $I_{a d v}$. The network predicts a totally black mask input $I_{a d v}$ while it predicts a roughly accurate mask input $I_{F}$.

denotes obtaining the gradients of our model when input $I_{F}$ and $L$ denotes the loss function. $\epsilon$ is taken a random number in the range $(0,0.01]$ in every iteration to increase randomness, which generates more training data during SAT and would make the network more robust. The model is then trained on the obtained adversarial example $I_{\text {adv }}$ and $\left.y_{[} g t\right]$, the corresponding ground-truth mask also used in the first training phase. We update the parameters $\theta$ of our network in Step 6 and Step 14 by back-propagation to minimize the loss function of Eq. 4

We draw a flowchart to explain the training phase in Fig. 7 The first phase is the traditional training process. In a single training iteration, we add a second phase to perform SAT. Specifically, after the normal back-propagation using the inputground-truth pairs and updating the network's parameters, the same inputs are used to generate adversarial samples Input $t_{a d v}$ by adversarial attack method, FGSM, according to the updated model. Although Input $_{a d v}$ has the same content as Input, which can be localized the tampered regions by the updated model, Input $_{a d v}$ is with adversarial samples and can mislead the detection result, shown in Fig. 6(b). In other words, Input $_{a d v}$ is new data for the model. This phase increases the training data dynamically since the adversarial samples are generated according to the updated model's parameters in every training iteration. Note that we optimize the same network in both the first and the second training phase.

With two-phase self-adversarial training, SAT can provide new training data dynamically from limited original samples. Those training data generated via adversarial attacks, e.g. FGSM, can make our model more robust.

To illustrate the impacts of SAT, we draw the residual map between $I_{F}$ and $I_{\mathrm{adv}}$ and then enlarge it by 20 times, as shown it in Fig. 6.a). The residual map will change in the following epoch because $I_{\mathrm{adv}}$ is constantly changing. Meanwhile, to differ the forged image $I_{F}$ and its corresponding adversarial example $I_{\text {adv }}$, we illustrate the inference results of them before training $I_{\text {adv }}$ in Fig. 6(b). As shown in Fig. 6(b), before the second training phase, the model predicts an approximately precise mask using $I_{F}$ but predicts a wrong mask using $I_{\text {adv }}$.

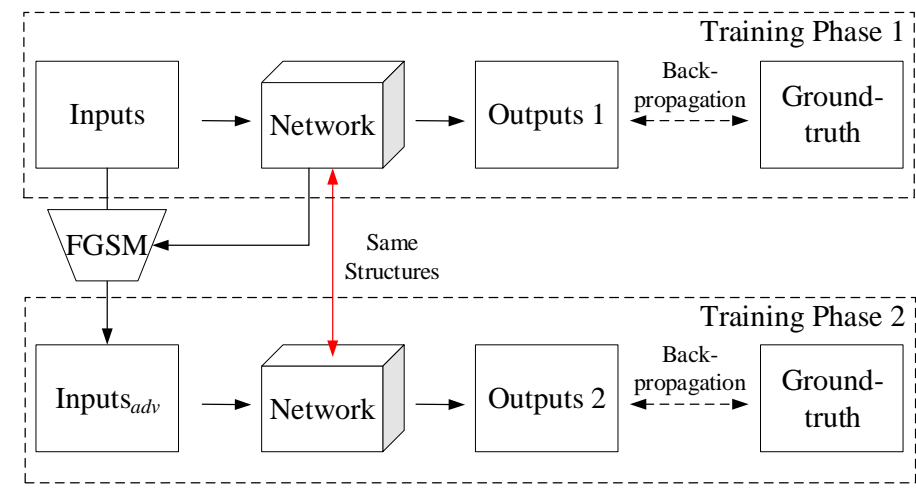

Fig. 7. The flowchart of our training phase.

\section{F. Visualization Analysis}

To explore the internal mechanism of our proposed CWHPF and forgery attention, visualization analysis was conducted in this section. As illustrated in Fig. 8, a forged image was taken as examples. We visualized the activation maps of different noise features extracted from a normal high pass filter layer [11] and our proposed CW-HPF. CW-HPF uses the inter-channel information while HPF does not. Then in Figs. 9 and 10 , we visualized the activation maps of different attention feature maps predicted by a normal attention module [23] and our proposed forgery attention. The activation maps with heatmaps were super-imposed on the forged image, where the red regions were with high activation values while the blue ones were with low values.

1) $C W-H P F$ : As we can see in Fig. 8, the flower (fake), the rock (pristine) and the birds (pristine) all naturally contain high-frequency information, which both normal HPF layer and our proposed CW-HPF can extract. However, we can see HPF extracts all high-frequency information while our proposed CW-HPF can just focus on the more precise noise features in the manipulated area by leveraging the interchannel relationships. As a result, the flower is much more prominent in the heatmap corresponding to our proposed CWHPF. 


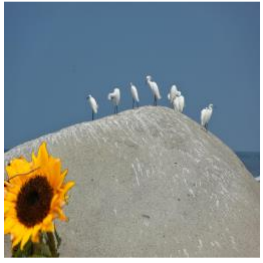

Forgery

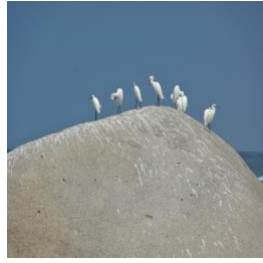

Original Image

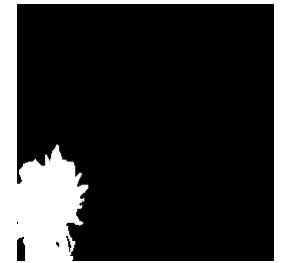

Groundtruth Mask

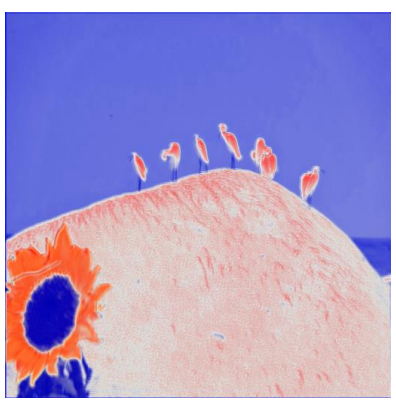

HPF Noise Features

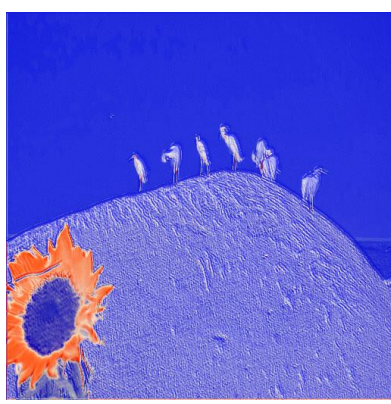

CW-HPF Noise Features $\sigma^{2}=0.4 \times 10^{-4}$
Fig. 8. Visualization of different noise features extracted from an example image (NIST2016_4867.jpg) from NIST. From the visualization we can observe that HPF noise features are highly responsive at rock and birds in red, which are pristine regions, while ours are not. It indicates that CW-HPF noise features respond weakly to pristine regions but more intensive on tampered regions.

The variance of each noise feature map was also calculated. As shown in Fig. 8, the variance $\sigma^{2}$ of our CW-HPF noise features is only one-ninth of that of HPF noise features, which also reveals that CW-HPF noise features have a smaller internal gap. The small variance enables CW-HPF to extract more consistent noise features in the tampered regions.

One possible reason of this phenomenon is that our CWHPF takes advantage of inter-channel relationships to enhance the correlation between all channels. These relationships amplify the slight perturbations of noise inconsistency. Therefore $\mathrm{CW}-\mathrm{HPF}$ focuses more on the tampered regions.

2) Forgery Attention: Figs. 9 and 10 visualize different regional attention of normal attention features and our proposed forgery attention features. Especially in Fig. 10, the redder regions in the heatmap of normal attention features fell in two persons while forgery attention had higher responses to the background. It indicates that the forgery attention focuses more on the tampered regions instead of the salient objects. That's because architectures of normal attention modules are designed to detect the texture of objects. On the contrary, our forgery attention aims to pay more attention to the tampered traces.

The possible reason why forgery attention aims to pay more attention to the tampered traces is that it explores global contextual noise information by building associations among noise features with the attention mechanism. In addition, it can also be attributed to CW-HPF which extracts the noise features first. Our method can adaptively aggregate long-term contextual information of noise features, thus improves feature representation for forgery localization.

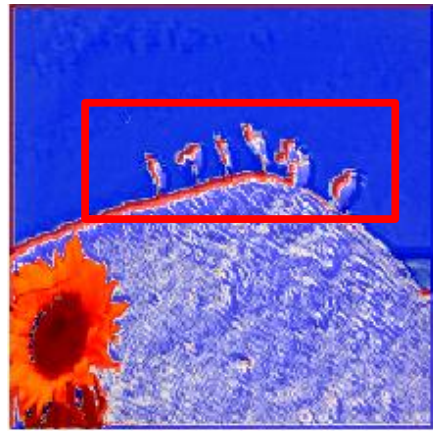

Attention Feature

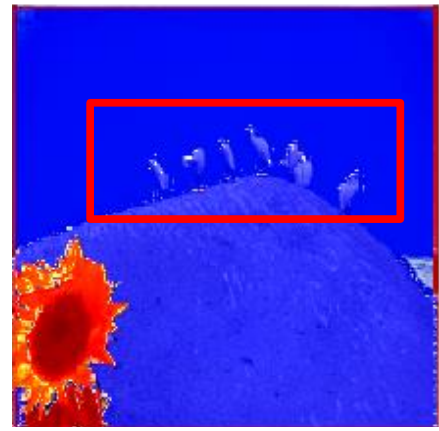

Forgery Attention Feature
Fig. 9. Visualization of different attention features of the example image in Fig. 8 The red box emphasizes untampered objects, namely the birds. The untampered area of the left heatmap is redder than that of the right one, which indicates that the normal attention module responds to all objective regions while our forgery attention focuses more on the tampered regions.

Specifically, two attention branches contribute to focusing on noise interdependencies. It can be inferred from Equation 1 that the resulting feature $\mathrm{SAF}_{N}$ at each position is a weighted sum of the noise features across all pixels and the original feature, which gives it a global contextual view and selectively aggregative contexts. Similar noise features achieve mutual gains, thus improving the noise consistency between the tampered and original regions. Furthermore, Equation 2 shows that the final feature of each channel is a weighted sum of the noise features of all channels and original features, which further models long-term noise dependencies among feature maps to boost noise feature discriminability. Each highlevel channel can be regarded as a class-specific response, and different noise responses are associated with each other. Exploiting the interdependencies between channel maps emphasizes interdependent feature maps and improves the feature representation of tampered traces. Therefore, forgery attention achieves better performance in forgery localization tasks.

\section{EXPERIMENTS}

In this section, we carry out comprehensive experiments to demonstrate our proposed approach on several benchmark datasets and compare the results with state-of-the-art methods. Besides, we evaluate the robustness of our method in the scenarios of resizing, JPEG compression, and adversarial attacks. All of our experimental results are obtained on realworld datasets under the actual hardware experiments. There are no numerical simulations in our experiments. The source codes and auxiliary materials are available for download from GitHub 1

\section{A. Setup}

1) Datasets: The following datasets are used in our experiments:

- DEFACTO [25] is a synthesized dataset generated from MSCOCO [34]. Three typical types of forgeries (i.e.,

${ }^{1}$ https://github.com/tansq/SATFL 
TABLE I

QUANTITATIVE RESULTS OF ABLATION STUDIES ON DEFACTO

\begin{tabular}{ccc}
\hline Model & AUC & $F_{1}$ \\
\hline Baseline & 0.978 & 0.855 \\
CW-HPF Model & 0.986 & 0.878 \\
Forgery Attention Model & 0.990 & 0.890 \\
\hline Coarse-to-fine Forgery Attention Model & 0.992 & 0.904 \\
Coarse-to-fine Forgery Attention Model+SAT & 0.996 & 0.920 \\
\hline Coarse-to-fine Forgery Attention Model+SAT+Flipping+Rotation & $\mathbf{0 . 9 9 8}$ & $\mathbf{0 . 9 2 9}$ \\
\hline
\end{tabular}

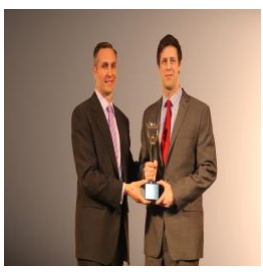

Forgery

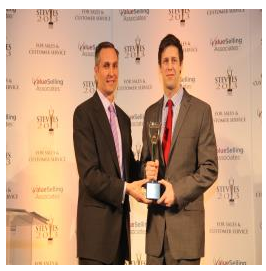

Original Image

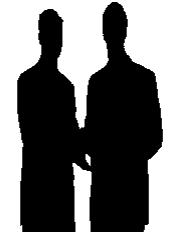

Groundtruth Mask

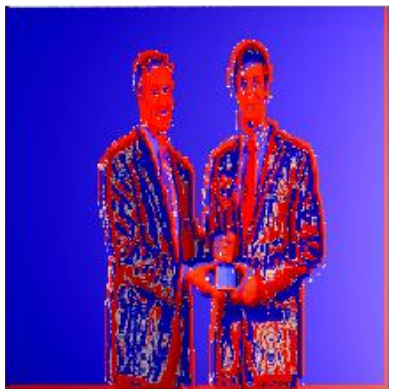

Attention Feature

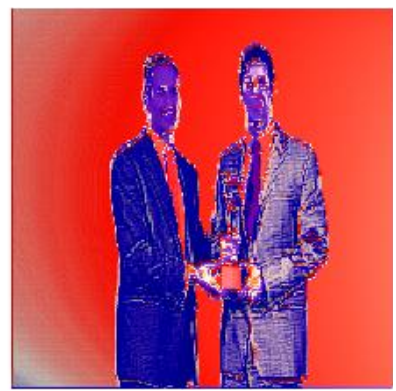

Forgery Attention Feature
Fig. 10. Visualization of different attention features of an example image (NIST2016_3119.jpg) from NIST 2016. The normal attention module responds to the objects (i.e., persons) in red while our forgery attention responds more to the tampered regions (the background).

splicing, copy-move, and removal) are involved in DEFACTO. 98,779 tampered images are selected from DEFACTO as our base dataset for ablation study and pretraining. We have to emphasize that our base dataset contains fewer images than other studies', such as MantraNet [4] (102,028 samples) and SPAN [14] (102,028 samples). The training-testing ratio is set to $9: 1$.

- Columbia [35] provides 180 splicing images with edge masks. The ground-truth masks are generated by ourselves from the corresponding edge masks

- CASIA [27] contains splicing and copy-move images in which forged regions are carefully selected. Some forged images have been further post-processed with filtering and blurring. It can be split into CASIA $2.0(5,123$ samples) for training and CASIA 1.0 (921 samples) for testing. Both of them are provided with ground-truth masks.

- COVERAGE [9] contains 100 forged images manipulated by copy-moving. All the images are post-processed to remove visual traces. It is provided with ground-truth masks.

- NIST [26] is composed of 564 samples manipulated with splicing, copy-move, or removal. The visible traces of manipulations are concealed by post-processing. The dataset has ground-truth masks for evaluation.

- PS-dataset is a large-resolution dataset introduced in the latest work [15]. It involves three sub-datasets, namely PS-boundary dataset, PS-arbitrary dataset and PS-script dataset. Among these sub-datasets, the PS-boundary dataset and PS-arbitrary dataset are created manually with Photoshop ${ }^{\circledR}$, while the PS-script dataset is tampered with the automatic script.

Please note that for the sake of fair comparison, our experiments follow the training-testing ratio configuration in RGB$\mathrm{N}$ [11] on NIST, COVERAGE, and CASIA.

2) Implementation Details: Our approach has been implemented based on TensorFlow. Our framework was trained with input images resized to $512 \times 512$ on a single Tesla P100 GPU ${ }^{2}$ An ADAM solver was used to optimize the model with a learning rate of 0.002 .

3) Evaluation Metrics: Pixel-level $F_{1}$ score and Area Under the receiver operating Curve (AUC) were employed as our evaluation metrics. $F_{1}$ score and AUC measured the performance of binary classification for every pixel, where higher scores indicate better performance. Both pixel-level AUC and $F_{1}$ score values range in [0,1]. Kindly note according to observations based on our experimental results, $F_{1}$ score is more accurate than AUC. This is due to the fact that $F_{1}$ score drops significantly when the predicted masks contain quite a few false-positive predictions (the pristine pixels are marked as tampered). On the contrary, AUC still remains at a high score on account of some factors such as threshold settings in this case.

4) Loss Function: During training, binary cross-entropy loss was used as our training loss function. It was minimized by optimizing the model parameters. The loss function is formulated as follows:

$$
\text { Loss }=L_{\mathrm{BCE}}\left(y_{\mathrm{gt}}, y_{M_{1}}\right)+L_{\mathrm{BCE}}\left(y_{\mathrm{gt}}, y_{M}\right),
$$

where $L_{\mathrm{BCE}}$ denotes Binary Cross-Entropy loss, $y_{\mathrm{gt}}$ denotes ground-truth masks, $y_{M_{1}}$ denotes coarse masks, and $y_{M}$ denotes refined masks.

\footnotetext{
${ }^{2}$ OpenCV package was used to resize the images. We set interpolation=INTER_AREA when resizing the forged images and set interpolation=INTER_NEAREST for resizing the masks.
} 
5) Compared Methods: We have selected several advanced methods compared with our approach. The advanced methods can be categorized into two types, namely unsupervised methods and deep-learning based methods. The unsupervised methods [36], [37], [17] leverage feature extraction techniques to seek unnatural traces. Recently, the deep-learning based methods [13], [11], [4], [14] have demonstrated superior performance by using convolutional neural networks. In our work, we have compared our approach with these two types of methods.

\section{B. Ablation Study}

We have conducted extensive ablative experiments to validate each component of our framework. In particular, we have first generally conducted the progressive ablation study and then carefully conducted detailed experiments for each of the proposed components. All of the ablative experiments were conducted on the DEFACTO dataset.

1) Progressive Ablation Study: The progressive ablative experiments were conducted to validate our proposed components and SAT. The setup models were as follows:

- Baseline: The baseline model contained an HPF filter layer, a dilated convolutional module, and VGG blocks. It did not have a coarse-to-fine architecture.

- CW-HPF Model: The HPF filter layer was replaced in the baseline model with a CW-HPF filter block. Others remained the same.

- Forgery Attention Model: It was constructed based on the CW-HPF model, composed of CW-HPF, VGG blocks, a dilated convolutional module, and a forgery attention module.

- Coarse-to-fine Forgery Attention Model: A coarse-tofine net was constructed using the CW-HPF model as the coarse net and forgery attention model as the refined net. This is actual our proposed model.

All models were trained using the same setting, and the results are reported in Table II From Table I] it can be seen that our proposed components are all effective and improve the AUC and $F_{1}$ scores significantly. The CW-HPF block improves the performance by 0.008 in AUC and 0.023 in $F_{1}$. The forgery attention module further improves by 0.004 in AUC and 0.012 in $F_{1}$ over the CW-HPF model. The coarseto-fine architecture further improves by 0.002 in AUC and 0.014 in $F_{1}$ over the forgery attention model.

To evaluate our proposed self-adversarial training strategy, data augmentation techniques were applied for the well-trained coarse-to-fine forgery attention model, namely our proposed SAT plus flipping and rotation. As also shown in Table II our proposed SAT further boosts the performance by 0.004 in AUC and 0.016 in $F_{1}$. SAT provides dynamic training data for more robust performance. For optimal performance, flipping and rotation were employed to further augment the training dataset and achieve 0.998 in AUC and 0.929 in $F_{1}$.

2) Ablation Study for Forgery Attention: We have applied four models that utilize different attention structures on top of basic CW-HPF model:

- CAM only: Only CAM was used as attention module;
TABLE II

QUANTITATIVE RESULTS OF ABLATION STUDIES FOR FORGERY ATTENTION STRUCTURES ON DEFACTO.

\begin{tabular}{ccc}
\hline Model & AUC & $F_{1}$ \\
\hline CW-HPF Model & 0.986 & 0.878 \\
\hline CAM only & 0.962 & 0.840 \\
PAM only & 0.984 & 0.856 \\
Dual Attention (Softmax) & 0.986 & 0.879 \\
Dual Attention (w/o CW-HPF) & 0.985 & 0.879 \\
\hline Forgery Attention Model & $\mathbf{0 . 9 9 0}$ & $\mathbf{0 . 8 9 0}$ \\
\hline
\end{tabular}

- PAM only: Only PAM was used as attention module;

- Dual Attention (Softmax): CAM and PAM were combined in parallel but with Softmax layer instead of Sigmoid layer.

- Dual Attention (w/o CW-HPF): An alternative dual attention [23] module was used without CW-HPF block;

- Forgery Attention Model: Our proposed forgery attention module.

As shown in Table [I] a single CAM or a single PAM slightly decreases the performance of the basic model. Combining CAM and PAM makes good use of spatial and channel dependencies to generate more precise results. Adopting Softmax instead of Sigmoid, results in decreased AUC and $F_{1}$ as well. Meanwhile, applying the common dual attention module [23] without CW-HPF cannot improve the performance. This ablative study clearly shows that our proposed attention architecture with dual attention module, Sigmoid layer and $\mathrm{CW}-\mathrm{HPF}$ is more adaptive to forgery localization task. Three intuitive reasons can be concluded for the effectiveness of our proposed dual attention module. Firstly, spatial and channelwise contextual dependencies both are important to distinguish the intrinsic inconsistency; Secondly, Softmax, which is often used as the last activation function of a neural network, is not suitable to replace Sigmoid in the intermediate layers of a deep-learning framework; Thirdly, CW-HPF can further provide richer noise features for attention modules, which contributes to focusing on the high-pass inconsistency between the pristine regions and tampered regions.

3) Ablation Study for SAT: SAT is proposed to alleviate the problem of limited training data. SAT exploits adversarial attacks in every training iteration and generates new training data dynamically, which guides our model to defend from adversarial attacks and achieve more robust performance. Unlike traditional data augmentation, our SAT can provide unlimited new adversarial training data according to model updating. The main difference between SAT and regular data augmentation, such as flipping, is that SAT is based on the model's parameters while others focus on the given data. We propose SAT to explore the possibility of augmenting data through model parameters instead of through data. Traditional data augmentation techniques do not conflict with our SAT but can further improve the performance with SAT. However, the common augmentation would not benefit for defense against the attacks. A simple augmentation flipping has been used in training our network on the DEFACTO dataset. As seen in 
TABLE III

QUANTITATIVE RESULTS OF FLIPPING+ROTATION AND SAT ON DEFACTO DATASET.

\begin{tabular}{ccc}
\hline Data Augmentation & AUC & $F_{1}$ \\
\hline Baseline & 0.992 & 0.904 \\
\hline Flipping + Rotation & 0.993 & 0.921 \\
After Gaussian Noise Attack & 0.819 & 0.757 \\
\hline SAT & 0.996 & 0.920 \\
After Gaussian Noise Attack & 0.915 & 0.826 \\
\hline Flipping + Rotation + SAT & 0.998 & 0.929 \\
After Gaussian Noise Attack & 0.937 & 0.903 \\
\hline
\end{tabular}

Table III. both SAT and Flipping + Rotation improved the performance of the baseline model. Based on the model with flipping and rotation, SAT can further increase the detection scores by 0.004 in AUC and 0.004 in $F_{1}$. However, SAT enables the network to decrease slightly while flipping and rotation do not when the forged images are imposed with Gaussian Noise attack. Specifically, the network trained with Flipping + Rotation degrades by $17 \%$ in AUC and $18 \%$ in $F_{1}$ after attack while the network trained with SAT only decreases by $8 \%$ in AUC and $10 \%$ in $F_{1}$. Since the adversarial attack used in SAT is a powerful attack technique, the network can be more robust when meeting other attacks.

4) Ablation Study for CW-HPF: The ablative experiments have been conducted to demonstrate the effectiveness of CWHPF with parallel spatial and channel attention modules. Specifically, seven different feature extraction strategies have been evaluated in our proposed coarse-to-fine forgery attention module as follows:

- RGB + RGB: there was no HPF layer in neither the coarse net nor the refined net;

- HPF + RGB: there was only a normal HPF layer [11] at the bottom of the coarse net;

- RGB + HPF: there was only a normal HPF layer at the bottom of the refined net;

- HPF + HPF: the normal HPF layers were adopted at the bottom of both the coarse net and the refined net;

- CW-HPF + HPF: it contained a CW-HPF block at the bottom of the coarse net and a normal HPF layer at the bottom of the refined net.

- HPF + CW-HPF: it contained a normal HPF layer at the bottom of the coarse net and a CW-HPF block at the bottom of the refined net;

- CW-HPF + CW-HPF: the CW-HPF blocks were adopted at the bottom of both the coarse net and the refined net.

As we can see in Table. IV] the proposed method with two CW-HPF modules outperforms other methods. Specifically, the method without a high-pass filters layer performs poorly in final results, with only 0.804 in AUC and 0.721 in $F_{1}$. Applying one HPF module in either the coarse net or the refined net improves the performance by $0.160-0.166$ in AUC and 0.123-0.129 in $F_{1}$. Introducing HPF in both the coarse net and the refined net improves the performance in a clear margin
TABLE IV

QUANTITATIVE RESULTS OF ABLATION STUDIES FOR CW-HPF STRUCTURES ON DEFACTO.

\begin{tabular}{ccc}
\hline Model & AUC & $F_{1}$ \\
\hline RGB + RGB & 0.804 & 0.721 \\
HPF + RGB & 0.970 & 0.850 \\
RGB + HPF & 0.964 & 0.844 \\
HPF + HPF & 0.977 & 0.856 \\
CW-HPF + HPF & 0.981 & 0.867 \\
HPF + CW-HPF & 0.984 & 0.869 \\
\hline CW-HPF + CW-HPF & $\mathbf{0 . 9 9 2}$ & $\mathbf{0 . 9 0 4}$ \\
\hline
\end{tabular}

by 0.174 in AUC and 0.135 in $F_{1}$ compared to the model with RGB + RGB. One possible reason is that the tampering traces are hidden in the high-frequency domain, and the highpass filters can detect them. Then, the model with a CW-HPF block in either the coarse net or the refined net boosts the performance of two HPF blocks by 0.004-0.006 in AUC and $0.011-0.013$ in $F_{1}$. Furthermore, our proposed model with two CW-HPF further increases the detection scores by 0.008 in AUC and 0.035 in $F_{1}$ compared to the model with a CWHPF in the refined net and achieves the best performance. It indicates that our proposed CW-HPF module enhances noise features and boosts performance in both the coarse net and the refined net.

5) Ablation Study for Dilated Convolutional Module: DFCN has used a series of dilated convolution layers to enlarge receptive fields to avoid learning features from narrow local regions. Thus following their approach, four dilated convolution layers are applied to inflate the kernels by inserting zeros between kernel elements with different dilation rates for extracting features with larger receptive fields. We follow DFCN's settings, and the dilation rates in the four dilated convolution layers are $2,4,8$, and 16 , respectively. The dilated convolution layers bridge the encoder and the corresponding decoder. There are two sub-nets in a coarse-to-fine manner. Here, we have conducted ablative experiments to validate the effectiveness of the dilated convolutional module.

To compare with our final model, the coarse-to-fine forgery attention model of the revision, with two dilated convolutional modules in both the coarse net and the refined net, we have modified the bridge of the encoders and the decoders of two sub-nets as follows:

- Dilated + Dilated: it contained two dilated convolutional modules in the coarse net and the refined net.

- w/o Dilated + w/o Dilated: it directly connected the encoders and decoders without any dilated convolutional module.

- Dilated + w/o Dilated: it included a dilated convolutional module in the coarse net and no dilated convolutional module in the refined net.

- w/o Dilated + Dilated: it consisted of a dilated convolutional module in the refined net and no dilated convolutional module in the coarse net.

As we can see in Table $\mathrm{V}$, without any dilated convolutional 
TABLE V

QUANTITATIVE RESULTS OF DIFFERENT SETTINGS OF DILATED CONVOLUTIONAL MODULE ON DEFACTO DATASET.

\begin{tabular}{ccc}
\hline Model & AUC & $F_{1}$ \\
\hline Dilated + Dilated & 0.996 & 0.920 \\
\hline w/o Dilated + w/o Dilated & 0.993 & 0.910 \\
Dilated + w/o Dilated & 0.990 & 0.907 \\
w/o Dilated + Dilated & 0.994 & 0.913 \\
\hline
\end{tabular}

TABLE VI

QUANTITATIVE RESULTS OF DIFFERENT SETTINGS OF CONNECTION ON DEFACTO DATASET.

\begin{tabular}{ccc}
\hline Model & AUC & $F_{1}$ \\
\hline Direct Connection & 0.709 & 0.322 \\
Feature Connection & 0.996 & 0.920 \\
\hline
\end{tabular}

modules, the model decreases the performance by 0.003 in AUC and 0.010 in $F_{1}$. Interestingly, if a dilated convolutional module is used in the coarse net, the model's performance declines slightly compared to that without a convolutional module. Meanwhile, the model with a dilated convolutional module in the refined net increases by 0.001 in AUC and 0.003 in $F_{1}$ compared to the model w/o Dilated + w/o Dilated, but decreases by 0.002 in AUC and 0.007 in $F_{1}$. It indicates that the coarse-to-fine network is required to generate the richer features in the refined net than in the coarse net, and applying the dilated convolutional modules in both the coarse net and the refined net improves the performance.

6) Ablation Study for Coarse-to-Fine Connection: Generally, referring to the coarse-to-fine manner, the connection from the coarse net to the refined net is to directly transmit the results of the coarse net as the inputs of the refined net. However, the result of the coarse net is a binary mask, which does not contain any semantics for refinement in forgery localization. Therefore, rather than using the final results of the coarse net as the connection, we deliver complete feature information generated by the last deconvolutional layer of the coarse net to the refined net, which enables the refined net to be optimized along with the features. We validate this design through an ablative experiment. We adopt the CW-HPF model as the coarse net and forgery attention model as the refined net and set two types of bridges between the coarse net and the refined net. The first type of connection uses the results of the coarse net, defined as Direct Connection, while the second one applies the output features of the last deconvolutional layer of the coarse net, defined as Feature Connection.

The results are shown in Table VI. The coarse-to-fine manner with Direct Connection shows a poor performance compared to the Feature Connection significantly. Specifically, the AUC of Feature Connection is $40 \%$ higher and the $F_{1}$ is $185 \%$ than Direct Connection. The possible reason is that Feature Connection provides integral features for the refined net while Direct Connection does not.

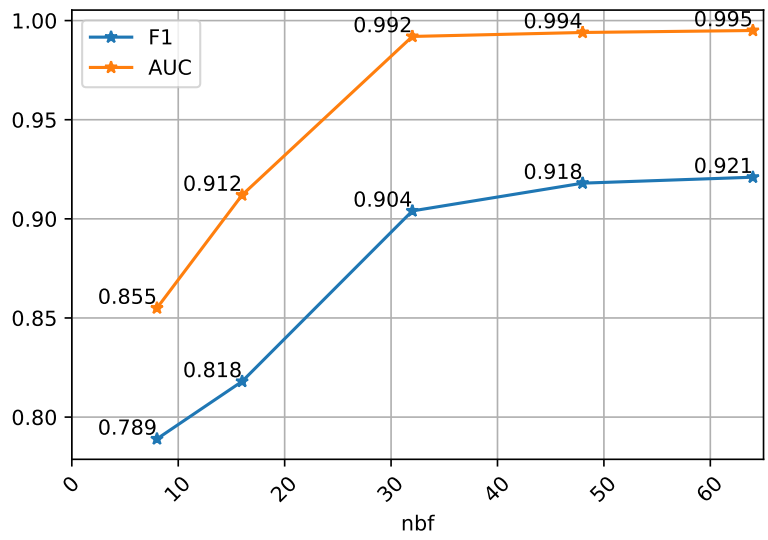

Fig. 11. The results of trade-off experiments.

7) Trade-off Experiments: We have conducted the tradeoff experiments based on the Coarse-to-fine forgery attention model to achieve a better trade-off. We employ different numbers of basic filters $(8,16,32,48,64)$ to conduct the trade-off experiments on the DEFACTO dataset. The different number of basic filters (nbf) represents the last deconvolutional layer's filter numbers while other filters of convolution layers in the network have multiples. The more filters indicate the larger parameters and cost, whereas the double nbf indicates the double network parameters and four calculation resources. Therefore, we gain our trade-off by this experiment.

The results are shown in Fig. 11. As we can see, there is a significant rise of $F_{1}$ score from 8 to 32 nbf while the raising speed slows down dramatically after 32 nbf. We can conclude that we achieve a better trade-off when applying 32 as the number of basic filters.

We have also added this experiment into the main text of the revised version in Section III B. For details, please refer to the corresponding context.

\section{Quantitative Results Compared against State-of-the-art Methods}

We compare the performance of our framework against several related methods on four benchmarks, namely NIST, COVERAGE, Columbia, and CASIA. The related methods include classic unsupervised methods, such as ELA [36], NOI1 [37], and CFA1 [17], and the latest deep networks, including H-LSTM [13], RGB-N [11], Mantra-Net [4], and SPAN [14]. We evaluated our framework under different setups: (1) benchmark training only; (2) fine-tuning. Under the benchmark training only setup, our model was trained on each benchmark separately. Under the fine-tuning setup, to achieve optimal performance, our pre-trained model was fine-tuned using several benchmarks, including NIST, COVERAGE, and CASIA, and tested on the corresponding testing split. Note that a training-testing ratio was set to 7:3 during benchmark training on the Columbia dataset. The actual reason has been given in accompanying discussions of Table VII.

The results are reported in Table VII. When adopting the benchmark training setup that uses only a small amount of 
TABLE VII

QUANTITATIVE RESULTS COMPARED AGAINST RELATED METHODS. — DENOTES THE RESULT IS UNAVAILABLE USING THE ORIGINAL METHOD AND * DENOTES WE USE THE DIFFERENT SETTINGS TO ADJUST OUR PRE-TRAINED METHOD TO COLUMBIA DATASET.

\begin{tabular}{cccccccccc}
\hline \multirow{2}{*}{ Method } & \multirow{2}{*}{ Training Method } & \multicolumn{2}{c}{ NIST } & \multicolumn{3}{c}{ COVERAGE } & \multicolumn{2}{c}{ Columbia } & \multicolumn{2}{c}{ CASIA } \\
\cline { 3 - 10 } & & AUC & $F_{1}$ & AUC & $F_{1}$ & AUC & $F_{1}$ & AUC & $F_{1}$ \\
\hline ELA & unsupervised & 0.429 & 0.236 & 0.583 & 0.222 & 0.581 & 0.470 & 0.613 & 0.214 \\
NOI1 & unsupervised & 0.487 & 0.285 & 0.587 & 0.269 & 0.546 & 0.574 & 0.612 & 0.263 \\
CFA1 & unsupervised & 0.501 & 0.174 & 0.485 & 0.190 & 0.720 & 0.467 & 0.522 & 0.207 \\
\hline H-LSTM & fine-tuning & 0.794 & - & 0.712 & - & - & - & - & - \\
RGB-N & fine-tuning & 0.937 & 0.722 & 0.817 & 0.437 & 0.858 & 0.697 & 0.795 & 0.408 \\
Mantra-Net & pre-training & 0.795 & - & 0.819 & - & 0.824 & - & 0.817 & - \\
SPAN & fine-tuning & 0.961 & 0.582 & 0.937 & 0.558 & 0.936 & 0.815 & 0.838 & 0.382 \\
\hline Ours & benchmark training & 0.943 & 0.622 & 0.856 & 0.526 & 0.917 & 0.891 & 0.788 & 0.384 \\
Ours & finetuning & $\mathbf{0 . 9 9 0}$ & $\mathbf{0 . 8 7 8}$ & $\mathbf{0 . 9 8 5}$ & $\mathbf{0 . 8 4 3}$ & $\mathbf{0 . 9 9 9 *}$ & $\mathbf{0 . 9 8 3 *}$ & $\mathbf{0 . 8 4 3}$ & $\mathbf{0 . 5 9 2}$ \\
\hline
\end{tabular}

training data, e.g., 75 forged images from COVERAGE, our model shows superior performance compared to state-of-theart approaches, especially on the Columbia dataset where our $F_{1}$ score outperforms all other methods. It indicates that our approach does not rely on large-scale training data to achieve decent performance, which shows the effectiveness of our approach. In the fine-tuning setup, our approach takes good advantage of large-scale training data. It can be concluded that our approach is further boosted by large-scale training data. In particular, our approach outperforms SPAN by 0.296 in $F_{1}$ score on NIST dataset and 0.048 in AUC on COVERAGE dataset. Note that because all of the forged regions in Columbia dataset are large while the forged regions in DEAFCTO are small ones. Thus, there is a domain gap between Columbia and DEAFCTO. So different from the settings of SPAN and Mantra-Net, our results on Columbia dataset are based on 30\% testing data and the other $70 \%$ data is used for finetuning. It is clear from Table VII that our model that is trained on DEFACTO dataset and finetuned on each benchmark datasets achieves state-of-the-art performance. The possible reason is that the proposed components and SAT training strategy work jointly and achieve optimal performance.

Furthermore, we compare the effectiveness of our model with the most recent algorithm, i.e., dense fully convolutional network (DFCN) [15]. We followed this setting [15] and used $512 \times 512$ image patches for training while full images for testing. Our model was trained using PS-script dataset and finetuned using only $10 \%$ forged images of several datasets, respectively, i.e., 100 samples in PS-arbitrary dataset, 100 samples in PS-boundary dataset and 56 samples in NIST dataset. Note that in the setting of [15], which is different from our experiments on NIST dataset in Table VII. NIST dataset is split into $512 \times 512$ patches. Note that DFCN aims to localize the tampered region with high-resolution while other advanced methods do not. Therefore, for a fair comparison, we make two settings on NIST dataset. When we compare our method with other advanced methods, we apply the typical pre-processing process that resizes the tampered images into
TABLE VIII

QUANTITATIVE RESULTS AGAINST THE MOST RECENT ALGORITHM, DFCN. OUR ALGORITHM WAS FINETUNED USING 100 SAMPLES IN PS-ARBITRARY DATASET, 100 SAMPLES IN PS-BOUNDARY DATASET AND 56 SAMPLES IN NIST DATASET, RESPECTIVELY.

\begin{tabular}{ccccccc}
\hline \multirow{2}{*}{ Method } & \multicolumn{2}{c}{ PS-boundary } & \multicolumn{2}{c}{ PS-arbitrary } & \multicolumn{2}{c}{ NIST } \\
\cline { 2 - 7 } & AUC & $F_{1}$ & AUC & $F_{1}$ & AUC & $F_{1}$ \\
\hline DFCN (w/o fine-tuning) & 0.90 & 0.61 & $\mathbf{0 . 9 1}$ & 0.57 & $\mathbf{0 . 6 3}$ & 0.20 \\
Ours (w/o fine-tuning) & $\mathbf{0 . 9 1}$ & $\mathbf{0 . 7 6}$ & 0.90 & $\mathbf{0 . 5 8}$ & 0.61 & $\mathbf{0 . 2 3}$ \\
\hline DFCN (fine-tuning) & 0.99 & 0.82 & 0.97 & 0.67 & 0.80 & 0.38 \\
Ours (fine-tuning) & 0.99 & $\mathbf{0 . 9 0}$ & $\mathbf{0 . 9 8}$ & $\mathbf{0 . 6 9}$ & $\mathbf{0 . 8 5}$ & $\mathbf{0 . 4 0}$ \\
\hline
\end{tabular}

$512 \times 512$ resolutions. When we compare our method with DFCN, we follow DFCN's setting and crop the tampered images in NIST dataset into $512 \times 512$ patches. It can be seen from Table VIII that the performance of our model outperforms DFCN on three datasets. Before fine-tuning in each benchmark dataset, our results are slightly lower than DFCN in AUC on PS-arbitrary and NIST datasets. However, our model has been over DFCN by 0.01 in AUC on PSboundary dataset. About $F_{1}$ score, our results are about $25 \%$ higher on PS-boundary dataset and 15\% higher on NIST than DFCN's. After fine-tuning on each dataset, our method has made a good improvement and outperforms DFCN on three datasets. In particular, our results achieve about $10 \%$ in $F_{1}$ score higher on PS-boundary dataset, slightly higher in AUC and $F_{1}$ score on PS-arbitrary dataset, and about $6 \%$ in AUC on NIST dataset than DFCN.

\section{Computational Complexity Analyses}

The computational complexities have been calculated and compared to the existing benchmarks with $512 \times 512$ NIST forged images as input in a single NVIDIA ${ }^{\circledR}$ Tesla $^{\circledR}$ P100 GPU card. As for the benchmarks, we adopt two popular methods, namely Mantra-Net [4] and SPAN [14], for comparison. 
TABLE IX

COMPUTATIONAL COMPLEXITY COMPARED WITH STATE-OF-THE-ART METHODS. * DENOTES THE TRAINING TIME OF ONE EPOCH ON NIST DATASET. "M" REPRESENTS MILLION, "MS" REPRESENTS MILLISECONDS AND “MINS" REPRESENT MINUTES.

\begin{tabular}{lcccc}
\hline Method & Params(M) & FLOPs(M) & Inference time(ms) & Training time (mins)* \\
\hline Mantra-Net & 3.80 & 7.58 & 392 & 16 \\
SPAN & 4.06 & 8.11 & 527 & 18 \\
\hline Our & 12.31 & 31.26 & 126 & 8 \\
\hline
\end{tabular}

The analytic report can be found in Table IX in which the inference time is the average over randomly selected 1,000 samples, and the training time if the average over 20 epochs on NIST dataset.

Please note that for deep-learning image forgery localization models, average training time as well as inference time with every input images are the better metrics rather than model parameters and FLOPs, since quite a few existing approaches have adopted complex training/inference tricks which cannot be measured with only model parameters and FLOPs.

From Table [IX we can see that compared with MantraNet and SPAN, our proposed framework consumes much less training time as well as inference time, though our framework is with more model parameters and FLOPs. Our framework takes only roughly $126 \mathrm{~ms}$ per image on a single Tesla P100 GPU. This is due to the fact that our proposed framework is trained and validated in a fully end-to-end manner, while Mantra-Net and SPAN are with quite a few extra off-model time-consuming operations/calculations, such as the nestedand-sliding window based feature extractor, a large number of matrix operations.

\section{E. Qualitative Results}

In Fig. 12, we show the prediction masks of our proposed framework for some selected images. From a standalone testing set, we select six tampered images which are generated with three popular tampering techniques, including splicing, copy-move and removal, from the mentioned datasets. As shown in Fig. 12, in the tampered images the original semantics has been changed and consequently, a considerable understanding gap is caused. For example, the left splicing image added a stop sign on the road, damaging the autodriving system. However, our algorithm can localize their forged regions credibly. From Fig. 12, it can be seen that our approach produces accurate results against different tampering techniques. No matter whether they are tampered objects or background without recognizable objects such as snow, our method detects them with high precision. In summary, our method makes good use of spatial and channel-wise attention to noise features and can precisely spot tampering areas that are obvious or even indistinguishable to human beings.

\section{F. Robustness Experiments}

Robustness experiments of our framework have been conducted in this section. OpenCV built-in functions (including
AREAResize, GaussianBlur, GaussianNoise, and JPEGCompress) and adversarial attacks (FGSM) were employed to generate content-preserving manipulations on NIST. Note that epsilons of FGSM used in our SAT strategy were valued from 0 to 0.01 while the epsilon was 0.02 in the testing stage, which is a fair comparison. As shown in Table $\mathrm{X}$, our framework is quite immune to several types of attacks. All results except FGSM of SPAN are reported in SPAN [14].

\section{CONCLUSION}

In this paper, we propose a novel deep neural network solution and a self-adversarial training strategy to effectively localize tampered regions in an image. The major contributions of our work areas follows:

- We have proposed a novel attention mechanism adapting to forgery localization task, named forgery attention which can be used to effectively capture noise feature dependencies in both spatial and channel dimensions.

- We have presented a novel self-adversarial training strategy for forgery localization, which augments training data dynamically to enable our model to achieve more robust performance, and alleviates the problem of limited labeled training data in this scenario.

- We have proposed a novel forgery localization framework in a coarse-to-fine manner, equipped with the ChannelWise High Pass Filter (CW-HPF) block. Extensive experiments conducted on de-facto benchmarking datasets demonstrate that our approach outperforms other stateof-the-art solutions in the literature by a clear margin.

Our future work will mainly focus on two aspects: (1) introduction of few-shot learning and even unsupervised learning based strategies to further tackle the issue of limited training data; (2) further exploration of the feasibility of our proposed approach in the wider multimedia forensics applications, e.g., video forgery localization and deepfake detection.

\section{REFERENCES}

[1] L. Verdoliva, "Media forensics and deepfakes: An overview," IEEE Journal of Selected Topics in Signal Processing, vol. 14, no. 5, pp. 910-932, 2020.

[2] T. M. Ghanim and A. M. Nabil, "Offline signature verification and forgery detection approach," in Proceedings of the 13th International Conference on Computer Engineering and Systems, 2018, pp. 293-298.

[3] E. M. Bik, A. Casadevall, and F. C. Fang, "The prevalence of inappropriate image duplication in biomedical research publications," mBio, vol. 7, no. 3, 2016. [Online]. Available: https://mbio.asm.org/ content/7/3/e00809-16

[4] Y. Wu, W. AbdAlmageed, and P. Natarajan, "Mantra-net: Manipulation tracing network for detection and localization of image forgeries with anomalous features," in Proceedings of the IEEE Conference on Computer Vision and Pattern Recognition, 2019, pp. 9543-9552.

[5] G. Muhammad, M. Hussain, and G. Bebis, "Passive copy move image forgery detection using undecimated dyadic wavelet transform," Digital Investigation, vol. 9, no. 1, pp. 49-57, 2012.

[6] C.-M. Pun, X.-C. Yuan, and X.-L. Bi, "Image forgery detection using adaptive oversegmentation and feature point matching," IEEE Transactions on Information Forensics and Security, vol. 10, no. 8, pp. 17051716, 2015.

[7] M. Huh, A. Liu, A. Owens et al., "Fighting fake news: Image splice detection via learned self-consistency," in Proceedings of the European Conference on Computer Vision, 2018, pp. 101-117.

[8] R. Salloum, Y. Ren, and C.-C. J. Kuo, "Image splicing localization using a multi-task fully convolutional network (MFCN)," Journal of Visual Communication and Image Representation, vol. 51, pp. 201-209, 2018. 
TABLE X

ROBUSTNESS ANALYSIS OF OUR FRAMEWORK ON THE NIST DATASET. THE RESULTS ARE REPORTED IN PIXEL-LEVER AUC.

\begin{tabular}{cccc}
\hline Manipulations & Mantra-Net & SPAN & Ours \\
\hline None & 0.795 & 0.8395 & 0.990 \\
\hline Resize (0.78x) & 0.7743 & 0.8324 & 0.984 \\
Resize (0.25x) & 0.7552 & 0.8032 & 0.979 \\
\hline GaussianBlur (kernel size=3) & 0.7746 & 0.8310 & 0.983 \\
GaussianBlur (kernel size=5) & 0.7455 & 0.7915 & 0.951 \\
\hline GaussianNoise (sigma=3) & 0.6741 & 0.7517 & 0.937 \\
GaussianNoise (sigma=15) & 0.5855 & 0.6728 & 0.866 \\
\hline JPEGCompress (quality=100) & 0.7791 & 0.8359 & 0.978 \\
JPEGCompress (quality=50) & 0.7438 & 0.8068 & 0.938 \\
\hline FGSM (eps=0.02) & 0.5058 & 0.5401 & 0.986 \\
\hline
\end{tabular}
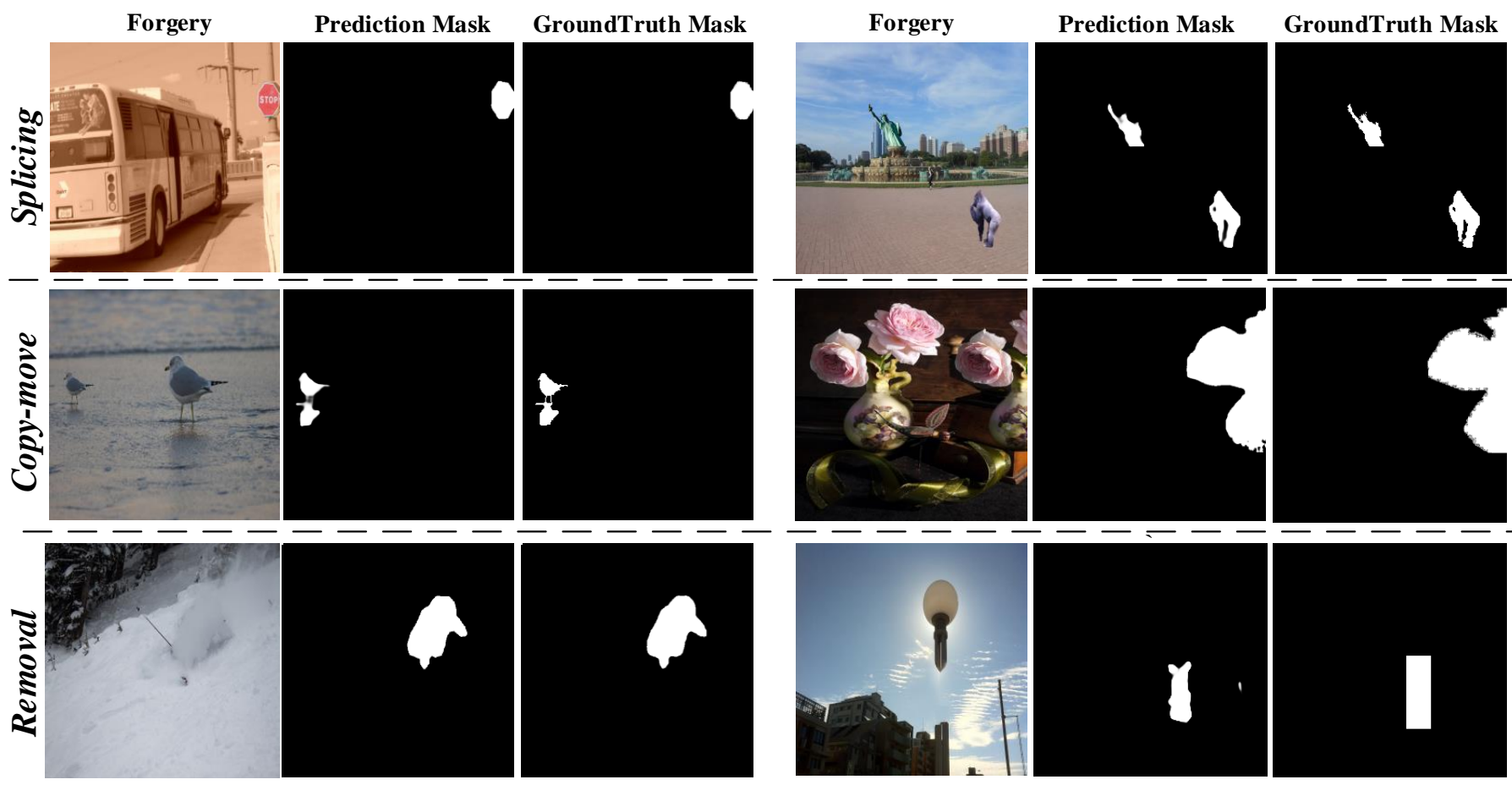

Fig. 12. Sample results of our framework in three popular manipulations, namely splicing, copy-move, and removal. The samples are from NIST, Columbia, COVERAGE, CASIA, and DEFACTO.

[9] B. Wen, Y. Zhu, R. Subramanian et al., "COVERAGE-a novel database for copy-move forgery detection," in Proceedings of IEEE International Conference on Image Processing, 2016, pp. 161-165.

[10] X. Zhu, Y. Qian, X. Zhao et al., "A deep learning approach to patchbased image inpainting forensics," Signal Processing: Image Communication, vol. 67, pp. 90-99, 2018.

[11] P. Zhou, X. Han, V. I. Morariu et al., "Learning rich features for image manipulation detection," in Proceedings of the IEEE Conference on Computer Vision and Pattern Recognition, 2018, pp. 1053-1061.

[12] J. H. Bappy, A. K. Roy-Chowdhury, J. Bunk et al., "Exploiting spatial structure for localizing manipulated image regions," in Proceedings of the IEEE International Conference on Computer Vision, 2017, pp. 49704979.

[13] J. H. Bappy, C. Simons, L. Nataraj et al., "Hybrid LSTM and encoderdecoder architecture for detection of image forgeries," IEEE Transactions on Image Processing, vol. 28, no. 7, pp. 3286-3300, 2019.
[14] X. Hu, Z. Zhang, Z. Jiang et al., "SPAN: Spatial pyramid attention network for image manipulation localization," in Proceedings of the European Conference on Computer Vision, 2020, pp. 312-328.

[15] P. Zhuang, H. Li, S. Tan et al., "Image tampering localization using a dense fully convolutional network," IEEE Transactions on Information Forensics and Security, vol. 16, pp. 2986-2999, 2021.

[16] T. Bianchi, A. De Rosa, and A. Piva, "Improved DCT coefficient analysis for forgery localization in JPEG images," in Proceedings of the IEEE International Conference on Acoustics, Speech and Signal Processing. IEEE, 2011, pp. 2444-2447.

[17] P. Ferrara, T. Bianchi, A. De Rosa et al., "Image forgery localization via fine-grained analysis of CFA artifacts," IEEE Transactions on Information Forensics and Security, vol. 7, no. 5, pp. 1566-1577, 2012.

[18] J. Fridrich and J. Kodovsky, "Rich models for steganalysis of digital images," IEEE Transactions on Information Forensics and Security, vol. 7, no. 3, pp. 868-882, 2012. 
[19] K. Simonyan and A. Zisserman, "Very deep convolutional networks for large-scale image recognition," arXiv preprint arXiv:1409.1556, 2014.

[20] A. Islam, C. Long, A. Basharat et al., "DOA-GAN: Dual-order attentive generative adversarial network for image copy-move forgery detection and localization," in Proceedings of the IEEE Conference on Computer Vision and Pattern Recognition, 2020, pp. 4676-4685.

[21] Y. Zhu, C. Chen, G. Yan et al., "AR-Net: Adaptive attention and residual refinement network for copy-move forgery detection," IEEE Transactions on Industrial Informatics, vol. 16, no. 10, pp. 6714-6723, 2020.

[22] J. Zeng, S. Tan, G. Liu et al., "WISERNet: Wider separate-thenreunion network for steganalysis of color images," IEEE Transactions on Information Forensics and Security, vol. 14, no. 10, pp. 2735-2748, 2019.

[23] J. Fu, J. Liu, H. Tian et al., "Dual attention network for scene segmentation," in Proceedings of the IEEE Conference on Computer Vision and Pattern Recognition, 2019, pp. 3146-3154.

[24] I. J. Goodfellow, J. Shlens, and C. Szegedy, "Explaining and harnessing adversarial examples," arXiv preprint arXiv:1412.6572, 2014.

[25] G. Mahfoudi, B. Tajini, F. Retraint et al., "DEFACTO: Image and face manipulation dataset," in Proceedings of the 27th European Signal Processing Conference, 2019, pp. 1-5.

[26] H. Guan, M. Kozak, E. Robertson et al., "MFC datasets: Largescale benchmark datasets for media forensic challenge evaluation," in Proceedings of the IEEE Winter Applications of Computer Vision Workshops, 2019, pp. 63-72.

[27] J. Dong, W. Wang, and T. Tan, "CASIA image tampering detection evaluation database," in Proceedings of the IEEE China Summit and International Conference on Signal and Information Processing, 2013, pp. $422-426$.

[28] A. Vaswani, N. Shazeer, N. Parmar et al., "Attention is all you need," in Advances in Neural Information Processing Systems, 2017, pp. 59986008.

[29] K. Xu, J. Ba, R. Kiros et al., "Show, attend and tell: Neural image caption generation with visual attention," in International Conference on Machine Learning, 2015, pp. 2048-2057.

[30] X. Li, W. Wang, X. Hu et al., "Selective kernel networks," in Proceedings of the IEEE Conference on Computer Vision and Pattern Recognition, 2019, pp. 510-519.

[31] A. Bochkovskiy, C.-Y. Wang, and H.-Y. M. Liao, "YOLOv4: Optimal speed and accuracy of object detection," arXiv preprint arXiv:2004.10934, 2020.

[32] A. Kurakin, I. Goodfellow, and S. Bengio, "Adversarial examples in the physical world," arXiv preprint arXiv:1607.02533, 2016.

[33] Y. Dong, F. Liao, T. Pang et al., "Boosting adversarial attacks with momentum," in Proceedings of the IEEE conference on Computer Vision and Pattern Recognition, 2018, pp. 9185-9193.

[34] T. Y. Lin, M. Maire, S. Belongie et al., "Microsoft coco: Common objects in context," in Proceedings of the European Conference on Computer Vision, 2014.

[35] T.-T. Ng, J. Hsu, and S.-F. Chang, "Columbia image splicing detection evaluation dataset," DVMM lab. Columbia Univ CalPhotos Digit Libr, 2009.

[36] N. Krawetz and H. F. Solutions, "A picture's worth," Hacker Factor Solutions, vol. 6, no. 2, p. 2, 2007.

[37] B. Mahdian and S. Saic, "Using noise inconsistencies for blind image forensics," Image and Vision Computing, vol. 27, no. 10, pp. 1497-1503, 2009.

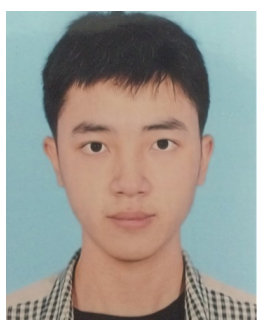

Long Zhuo received the B.S. degree in College of Computer Science and Software Engineering from Shenzhen University, Shenzhen, China in 2019. He is now at Sensetime Group. His current research interests include multimedia forensics, image generation and video generation.

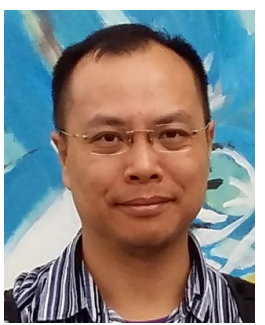

Shunquan Tan (M'10-SM'17) received the B.S. degree in computational mathematics and applied software and the Ph.D. degree in computer software and theory from Sun Yat-sen University, Guangzhou, China, in 2002 and 2007, respectively.

He was a Visiting Scholar with New Jersey Institute of Technology, Newark, NJ, USA, from 2005 to 2006. He is currently an Associate Professor with College of Computer Science and Software Engineering, Shenzhen University, China, which he joined in 2007. He is the Vice Director with the Shenzhen Key Laboratory of Media Security. His current research interests include multimedia security, multimedia forensics, and machine learning.

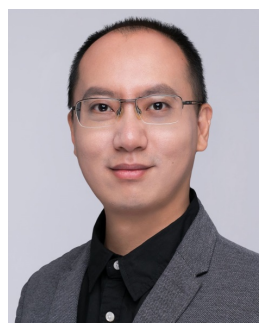

Bin Li (S'07-M'09-SM'17) received the B.E. degree in communication engineering and the Ph.D. degree in communication and information system from Sun Yat-sen University, Guangzhou, China, in 2004 and 2009, respectively.

He was a Visiting Scholar with the New Jersey Institute of Technology, Newark, NJ, USA, from 2007 to 2008. He is currently a Professor with Shenzhen University, Shenzhen, China, where he joined in 2009. He is also the Director with the Guangdong Key Lab of Intelligent Information Processing and the Director with the Shenzhen Key Laboratory of Media Security. He is an Associate Editor of the IEEE Transactions on Information Forensics and Security. His current research interests include multimedia forensics, image processing, and deep machine learning.

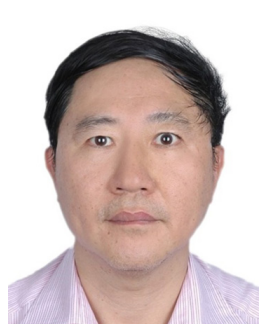

Jiwu Huang (M'98-SM'00-F'16) received the B.S. degree from Xidian University, Xi'an, China, in 1982, the M.S. degree from Tsinghua University, Beijing, China, in 1987, and the Ph.D. degree from the Institute of Automation, Chinese Academy of Science, Beijing, in 1998. He is currently a Professor with the College of Electronics and Information Engineering, Shenzhen University, Shenzhen, China. Before joining Shenzhen University, he has been with the School of Information Science and Technology, Sun Yat-sen University, Guangzhou, China, since 2000. His current research interests include multimedia forensics and security. He is an Associate Editor of the IEEE Transactions on Information Forensics and Security. 